\title{
On the absolute stability regions corresponding to partial sums of the exponential function
}

\author{
David Ketcheson* Tihamér A. Kocsis*† Lajos Lóczi* \\ January 10, 2018
}

\begin{abstract}
Certain numerical methods for initial value problems have as stability function the $n^{\text {th }}$ partial sum of the exponential function. We study the stability region, i.e., the set in the complex plane over which the $n^{\text {th }}$ partial sum has at most unit modulus. It is known that the asymptotic shape of the part of the stability region in the left half-plane is a semi-disk. We quantify this by providing disks that enclose or are enclosed by the stability region or its left half-plane part. The radius of the smallest disk centered at the origin that contains the stability region (or its portion in the left half-plane) is determined for $1 \leq n \leq 20$. Bounds on such radii are proved for $n \geq 2$; these bounds are shown to be optimal in the limit $n \rightarrow+\infty$. We prove that the stability region and its complement, restricted to the imaginary axis, consist of alternating intervals of length tending to $\pi$, as $n \rightarrow \infty$. Finally, we prove that a semi-disk in the left half-plane with vertical boundary being the imaginary axis and centered at the origin is included in the stability region if and only if $n \equiv 0 \bmod 4$ or $n \equiv 3 \bmod 4$. The maximal radii of such semi-disks are exactly determined for $1 \leq n \leq 20$.
\end{abstract}

*Supported by Award No. FIC/2010/05 - 2000000231, made by King Abdullah University of Science and Technology (KAUST). Email addresses: david.ketcheson@kaust.edu.sa, katihi@sze.hu, lajos.loczi@kaust.edu.sa.

${ }^{\dagger}$ Supported by TÁMOP-4.2.2.A-11/1/KONV-2012-0012: Basic research for the development of hybrid and electric vehicles. The Project is supported by the Hungarian Government and co-financed by the European Social Fund. 


\section{Introduction}

For a given positive integer $n$, consider the region

$$
\mathcal{U}_{n}:=\left\{z \in \mathbb{C}:\left|\sum_{k=0}^{n} \frac{z^{k}}{k !}\right| \leq 1\right\},
$$

i.e., the set in the complex plane over which the degree- $n$ Taylor polynomial of the exponential has at most unit modulus. These sets correspond to the region of absolute stability of some common numerical solvers for ordinary differential equations, including extrapolation methods, Taylor methods, and certain Runge-Kutta methods. Indeed, any one-step method for which the number of stages or derivatives used is equal to the order of accuracy must have as stability function the corresponding Taylor polynomial of the exponential.

It is convenient to introduce some preliminary notation first. Let

$$
\mathcal{P}_{n}(z):=\sum_{k=0}^{n} \frac{(n z)^{k}}{k !} \quad\left(n \in \mathbb{N}^{+}\right)
$$

denote the scaled $n^{\text {th }}$ partial sum of the exponential function, and let

$$
\mathcal{S}_{n}:=\left\{z \in \mathbb{C}:\left|\mathcal{P}_{n}(z)\right| \leq 1\right\}
$$

Many results can be stated more compactly by using $\mathcal{S}_{n}$ instead of $\mathcal{U}_{n}$. We refer to $\mathcal{U}_{n}$ as the unscaled region and $\mathcal{S}_{n}$ as the scaled region. Figures 1 and 2 show the boundaries of the first few unscaled and scaled stability regions, respectively.

By $D_{\varrho}\left(z_{0}\right)$ we mean the closed disk of radius $\varrho>0$ centered at $z_{0} \in \mathbb{C}$, and let $\Sigma_{1} \subset \mathbb{C}$ denote the Szegö region, which is depicted in Figure 3 and defined in Section 1.1. The boundary of the Szegö region, $\partial \Sigma_{1}$, is known as the Szegó curve. The closed left half of the complex plane is denoted by $\{\Re \leq 0\}$, the imaginary axis is $\{\Re=0\}$, and instead of $D_{\varrho}(0)$ we simply write $D_{\varrho}$.

We now recall some results from [8] regarding the shape of the scaled stability region for large enough $n$. Some sets from Theorems 1.1 and 1.3 are depicted in Figure 4.

Theorem 1.1 ([8], Lemma 5.1). For any $\varrho>0$ there exists a positive index $n_{0}(\varrho)$ such that

$$
\forall n \geq n_{0}(\varrho): \quad D_{\varrho}(-\varrho) \cap \Sigma_{1} \subset \mathcal{S}_{n}
$$

Theorem 1.2 ([8], Theorem 5.3). For any $0<\varrho<\frac{1}{2 e}$ there exists a positive index $n_{0}(\varrho)$ such that

$$
\forall n \geq n_{0}(\varrho): \quad D_{\varrho}(-\varrho) \subset \mathcal{S}_{n}
$$




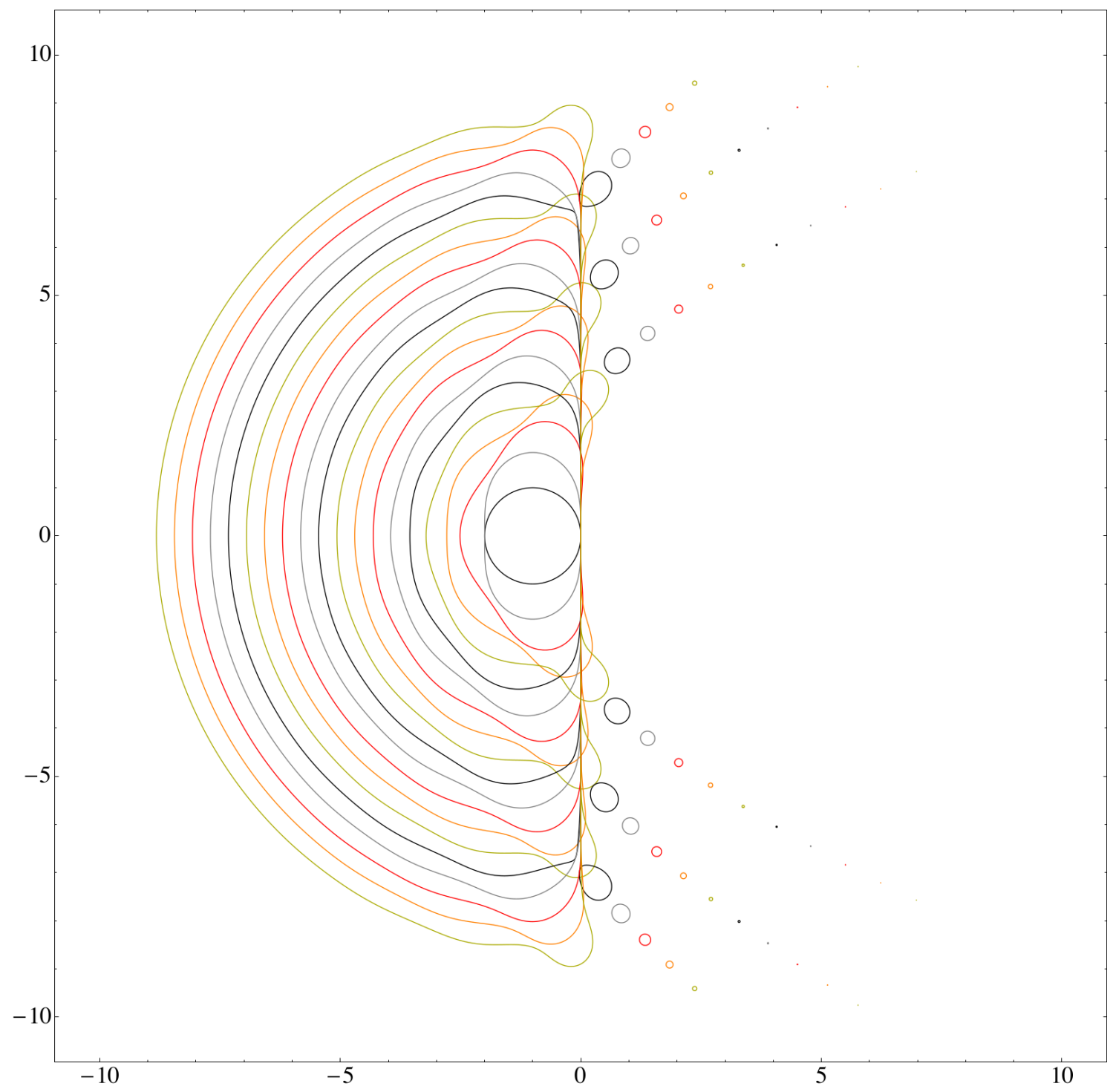

Figure 1: The boundary curve(s) of the unscaled stability regions $\mathcal{U}_{n}$ for $1 \leq n \leq 20$ in the square $-10 \leq \Re(z) \leq 10,-10 \leq \Im(z) \leq 10$. Five colors are used cyclically for different $n$ values. Compare this figure with Figure 7 (where 5 and 6 appear as certain block lengths) and Lemma 4.1 (which has "period 4"). 


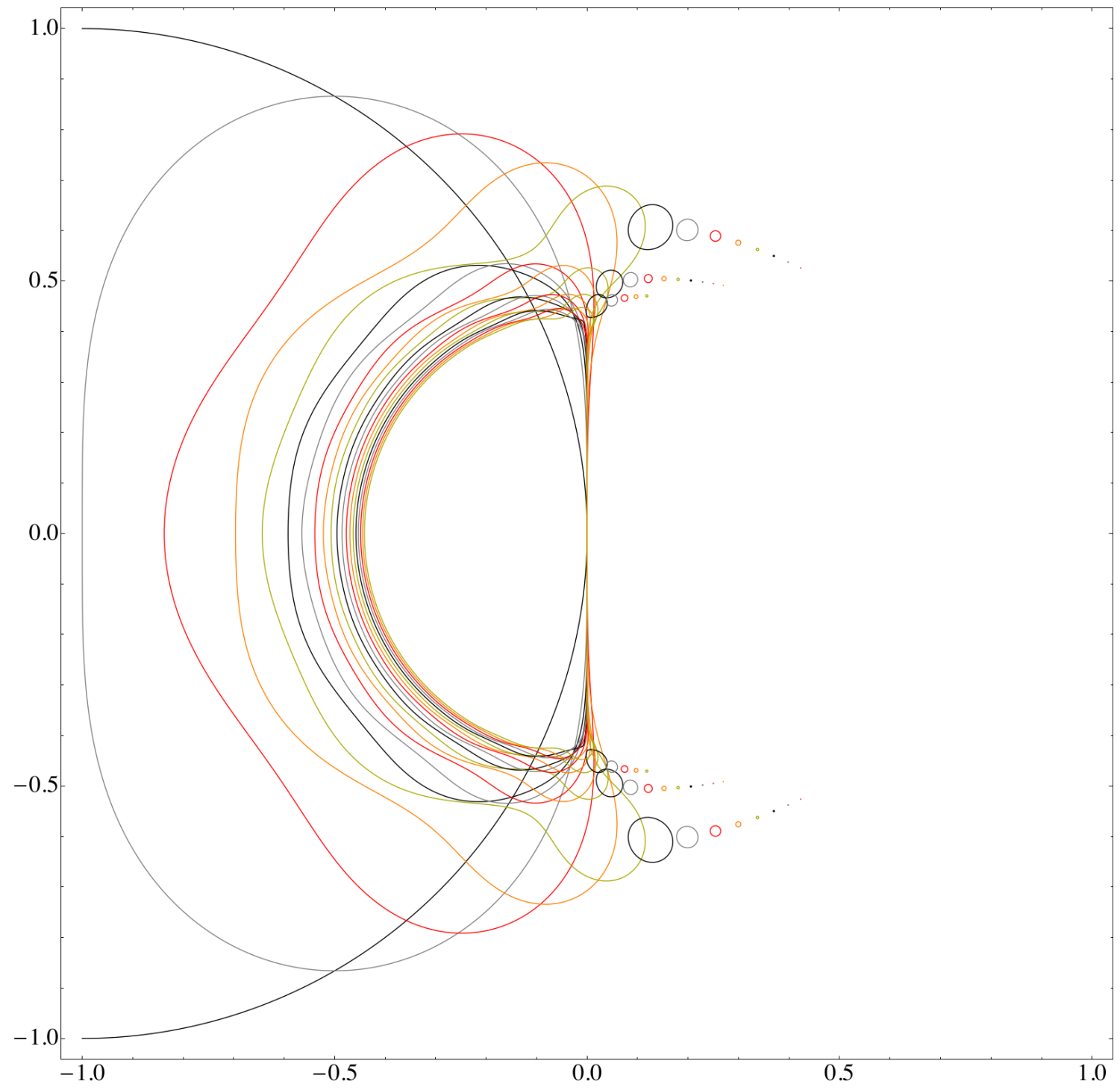

Figure 2: The boundary curve(s) of the scaled stability regions $\mathcal{S}_{n} \subset \mathbb{C}$ for $1 \leq n \leq 20$ in the square $-1 \leq \Re(z) \leq 1,-1 \leq \Im(z) \leq 1$. The color scheme is the same as that of Figure 1. Although the general number of connected components is not known, numerical investigations suggest that the set $\mathcal{S}_{n}$ is connected for $1 \leq n \leq 5$, has 3 connected components for $6 \leq n \leq 10$, and has 5 connected components for $n=11$, and so on; see also [7, pp. 73-74]. 
Theorem 1.3 ([8], Theorem 5.4). Let

$$
\begin{gathered}
\mathcal{S}_{\infty}:=\left\{z \in \mathbb{C}: \exists \text { a strictly increasing sequence } n_{k} \in \mathbb{N}^{+}(k=1,2, \ldots)\right. \text { and } \\
\left.\exists z_{n_{k}} \in \mathcal{S}_{n_{k}} \text { such that } \lim _{k \rightarrow+\infty} z_{n_{k}}=z\right\} .
\end{gathered}
$$

Then

$$
\mathcal{S}_{\infty}=\left(D_{1 / e} \cap\{\Re \leq 0\}\right) \cup \partial \Sigma_{1} .
$$

In practical numerical analysis, usually small values of $n$ are relevant. Hence the above theorems cannot be applied, since they do not specify the value of $n_{0}$. The direct motivation for the present work comes from [10], where inclusions of the form $\mathcal{U}_{n} \subset D_{\varrho_{n}}$ or $\mathcal{U}_{n} \cap\{\Re \leq$ $0\} \subset D_{\varrho_{n}} \cap\{\Re \leq 0\}$ with $\varrho_{n}>0$ as small as possible were needed for all (or large enough) $n \geq 1$ values. The primary aim of the present paper is to prove some results of this type.

The structure and main results of the paper are as follows. In Section 1.1 we establish notation, then in Section 1.2 we review some results relevant to our study. In Section 2 we prove the inclusion $\mathcal{S}_{n} \subset D_{1.6}$ for all $n \geq 2$, and, for any $0<\varepsilon<0.6$, the asymptotically optimal bound $\mathcal{S}_{n} \subset D_{1+\varepsilon}$ for $n \geq n_{0}(\varepsilon)$ large enough. In Section 3 we give similar bounds for the part of $\mathcal{S}_{n}$ that lies in the closed left half-plane: the constant 1.6 can be replaced by 0.95 in $\{\Re \leq 0\}$, and $1+\varepsilon$ by $1 / e+\varepsilon(\varepsilon>0)$. Sections 2 and 3 also contain exact values for the smallest $D_{\varrho_{n}}$ containing $\mathcal{S}_{n}$ and $\mathcal{S}_{n} \cap\{\Re \leq 0\}$, respectively, for $1 \leq n \leq 20$, based on direct computation. Section 4 contains some results regarding the boundary of $\mathcal{U}_{n}$ near the imaginary axis (for aesthetic reasons, we consider the boundary of $\mathcal{U}_{n}$ and not that of $\mathcal{S}_{n}$ ). In particular, we show that the slices $\mathcal{U}_{n} \cap\{\Re=0\}$ consist of alternating intervals and gaps, with the length of each interval and each gap converging to $\pi$ as $n \rightarrow+\infty$, and with offset depending on $n \bmod 4$. In Section 5 we prove that $D_{\varrho_{n}} \cap\{\Re \leq 0\} \subset \mathcal{S}_{n}$ for some $\varrho_{n}>0$ if and only if $n \equiv 0 \bmod 4$ or $n \equiv 3 \bmod 4$. We compute the largest such constants $\varrho_{n}$ for $1 \leq n \leq 20$. Finally, Section 6 contains the proofs of some technical lemmas required for the main results.

\subsection{Notation}

In the definition of $\mathcal{U}_{n}$ and $\mathcal{P}_{n}$, we use the usual convention that $0^{0}=1$.

The real and imaginary part of a complex number $z \in \mathbb{C}$ is denoted by $\Re(z)$ and $\Im(z)$, respectively. For $\delta \in \mathbb{R}$, by $\{\Re \leq \delta\}$ we mean $\{z \in \mathbb{C}: \Re(z) \leq \delta\}$; the definition of other similar symbols, such as $\{\Re>0\}$, is analogous.

The closed disk centered at $z_{0} \in \mathbb{C}$ with radius $\varrho>0$ is $D_{\varrho}\left(z_{0}\right)$; when $z_{0}=0$, we simply write $D_{\varrho}$. For any $\varrho>0$, the half circular arc in the left half-plane is denoted by $C_{\varrho}:=\{z \in \mathbb{C}:|z|=\varrho\} \cap\{\Re \leq 0\}$. 


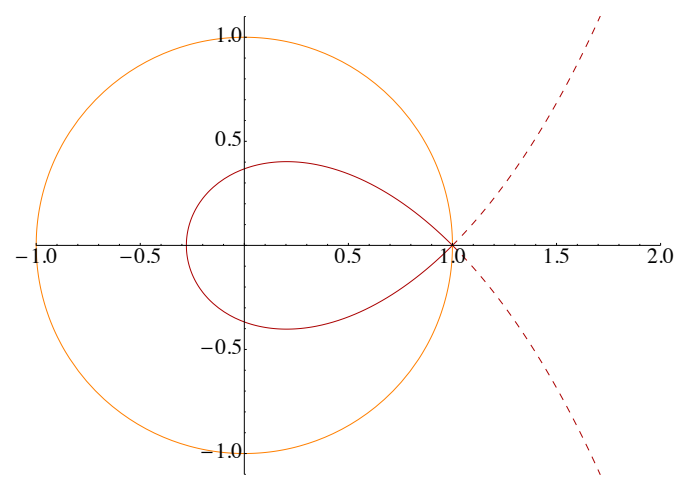

(a)

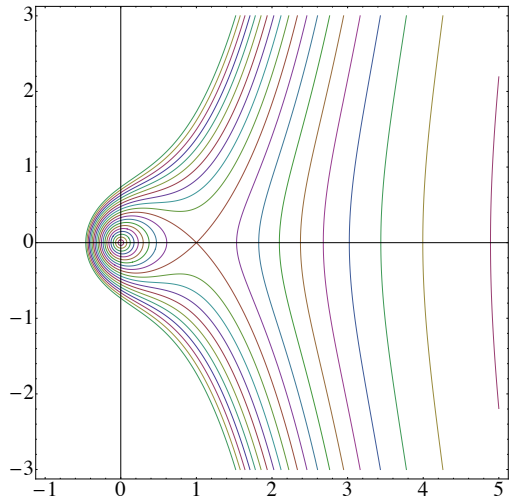

(b)

Figure 3: (a) The Szegő curve $\partial \Sigma_{1}$ as the boundary of the Szegö region in the unit disk; (b) some contour lines of $z \mapsto\left|z e^{1-z}\right|$.

The boundary of a bounded set $A \subset \mathbb{C}$ is denoted by $\partial A$.

We refer to the compact set

$$
\Sigma_{1}:=\left\{z \in \mathbb{C}:\left|z e^{1-z}\right| \leq 1\right\} \cap D_{1}
$$

as the Szegö region, with boundary, $\partial \Sigma_{1}$, known as the Szegő curve.

We use $|z, w|:=|z-w|$ to denote the distance between points $z, w \in \mathbb{C}$. Similarly, the distance between two sets $A, B \subset \mathbb{C}$ is $|A, B|:=\inf _{a \in A, b \in B}|a, b|$. When, for example, $A=\{z\}$ is a singleton, we simply write $|z, B|$ instead of $|\{z\}, B|$.

\subsection{Preliminaries}

For any $n \in \mathbb{N}^{+}$, let $\left\{\zeta_{k}(n)\right\}_{k=1}^{n}$ denote the zeros of $\mathcal{P}_{n}$. Szegö showed in his original paper [17] that $\zeta_{k}(n)$ cluster along the simple closed curve $\partial \Sigma_{1}$ as $n \rightarrow+\infty$. Many later works refined and extended this result; for example [5, 3, 12, 15, 16, 13, 4, 19, 27, 11, 9, 14, 20, 21, 25, 28, 1, 23, 22, 24].

Buckholtz gave some quantitative bounds on $\zeta_{k}(n)$ by showing that all zeros of $\mathcal{P}_{n}$ lie outside the Szegö region and that the zeros asymptotically approach the boundary of the Szegö region [3]. Specifically, for each $n$ and $1 \leq k \leq n$,

$$
\left|\zeta_{k}(n), \Sigma_{1}\right| \leq \frac{2 e}{\sqrt{n}}
$$




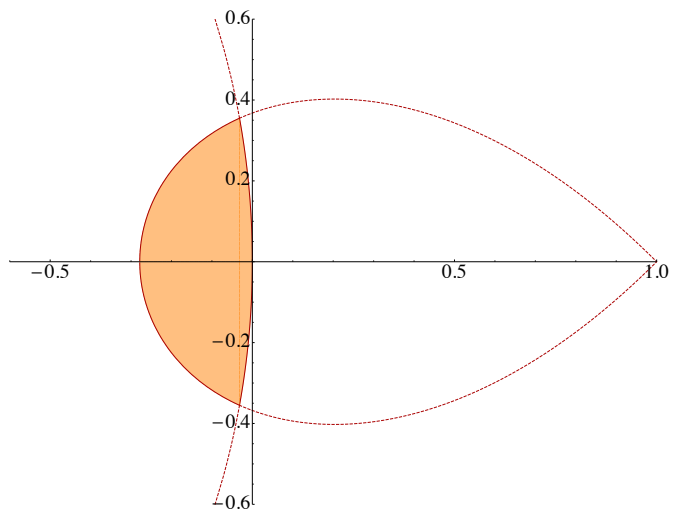

(a)

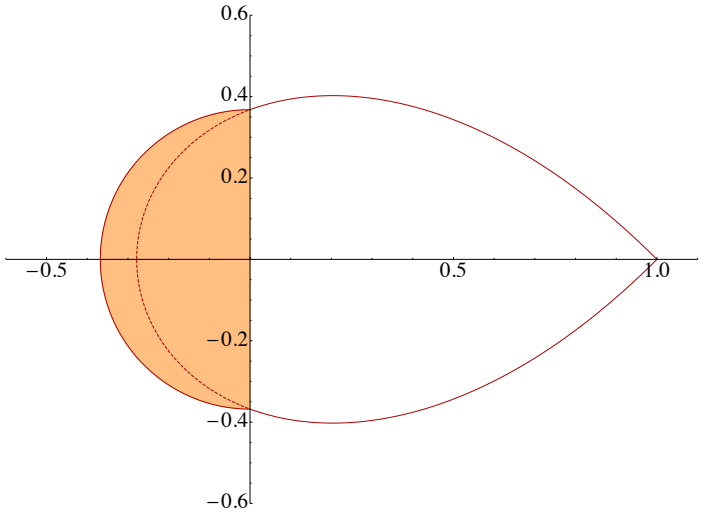

(b)

Figure 4: (a) The set $D_{2}(-2) \cap \Sigma_{1}$ from Theorem 1.1 (b) $\mathcal{S}_{\infty}$ from Theorem 1.3 .

The set $\mathcal{S}_{n}$ and the zeros of $\mathcal{P}_{n}$ are closely related; indeed, Theorems 1.1, 1.2 and 1.3 rely on the results of Szegő and Buckholtz regarding $\zeta_{k}(n)$.

Let us recall the main idea from [3, 2] about the proof of (1), since we are going to apply the same reasoning in Sections 2 and 3, but in a more quantitative way. Following [2], we define the function

$$
T_{n}(z):=\frac{n !}{(n z)^{n}} \mathcal{P}_{n}(z) \quad(z \in \mathbb{C} \backslash\{0\}),
$$

which satisfies the differential equation

$$
T_{n}(z)=\frac{z}{z-1}\left(1+\frac{T_{n}^{\prime}(z)}{n}\right) \quad(0 \neq z \neq 1)
$$

and the bounds

$$
\begin{gathered}
\forall z \in\left(\mathbb{C} \backslash \Sigma_{1}\right) \cup \partial \Sigma_{1}: \quad\left|T_{n}(z)\right| \leq 2 e \sqrt{n}, \\
\forall z \in \mathbb{C},|z| \geq 1: \quad\left|T_{n}(z)\right| \leq e \sqrt{n} ;
\end{gathered}
$$

see Figure 5. Recall the standard Cauchy inequality for the derivative:

Let $f$ be analytic for all $z \in D_{\delta}\left(z_{0}\right)$ and let $M=\max _{z \in D_{\delta}\left(z_{0}\right)}|f(z)|$. Then $\left|f^{\prime}\left(z_{0}\right)\right| \leq \frac{M}{\delta}$.

In [3], this inequality is used with (4) to estimate $\left|T_{n}^{\prime}\right|$ from above on $\left\{z \in \mathbb{C}:\left|z, \Sigma_{1}\right|>\right.$ $2 e / \sqrt{n}\}$, then with (3) to show $\left|T_{n}\right|>0$, and hence $\left|\mathcal{P}_{n}\right|>0$, on $\left\{z \in \mathbb{C}:\left|z, \Sigma_{1}\right|>2 e / \sqrt{n}\right\}$.

Finally, we also need the following explicit estimate, see, for example, [4]. 


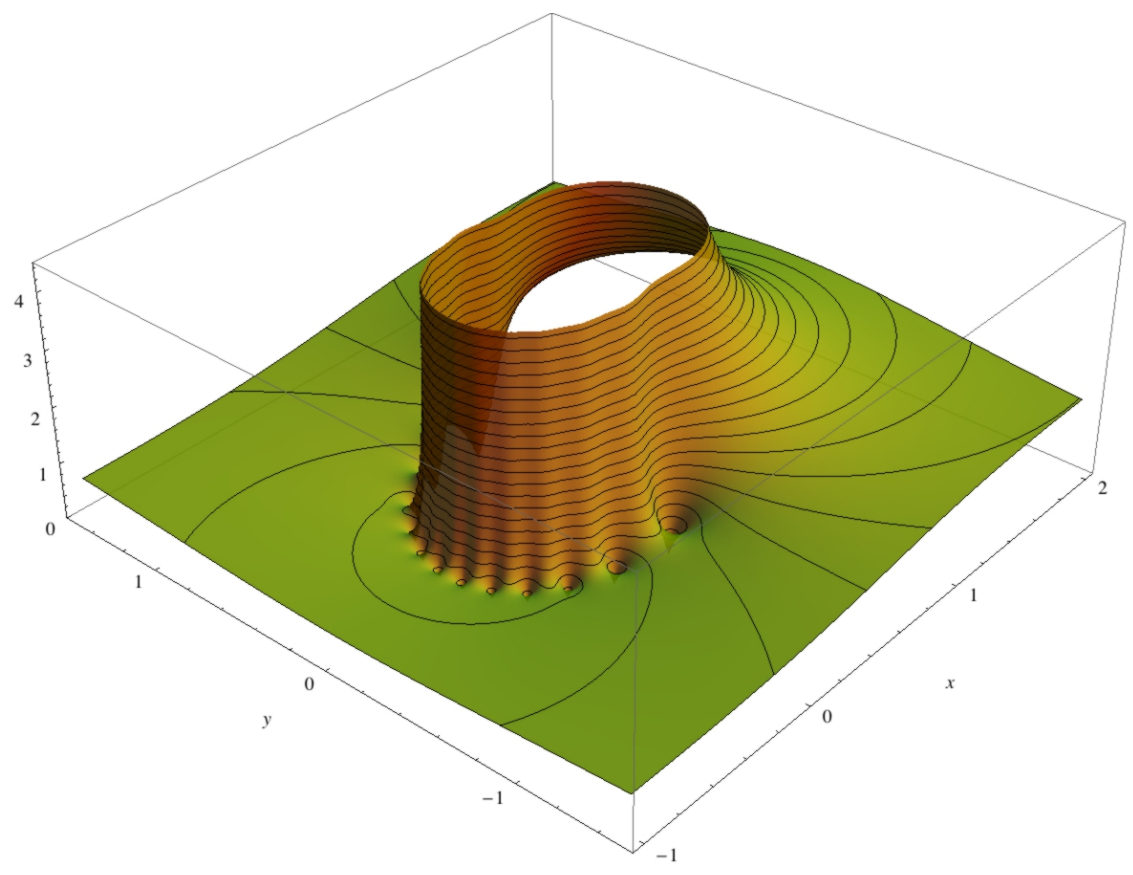

(a)

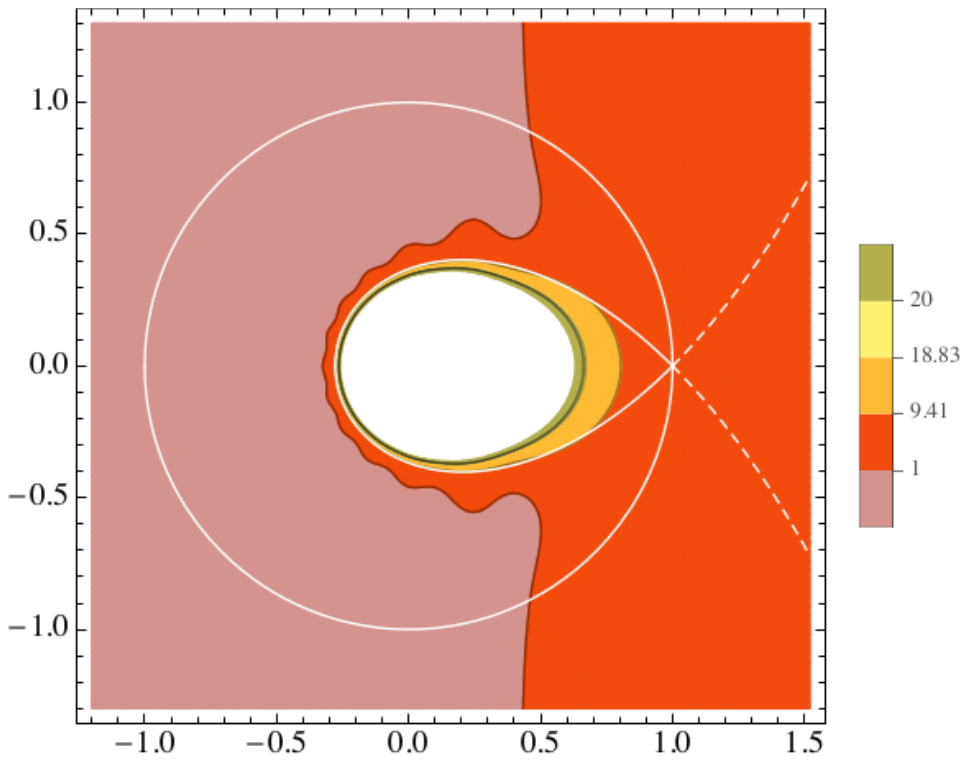

(b)

Figure 5: (a) Some level curves of $z \mapsto\left|T_{12}(z)\right|$ (defined by (2), and with $z=x+i y$ ); (b) $D_{1}$ and $\Sigma_{1}$ are displayed together with a contour plot of $\left|T_{12}\right|$ showing two specific contours at heights $e \sqrt{n}=e \sqrt{12} \approx 9.4$ and $2 e \sqrt{n}=2 e \sqrt{12} \approx 18.8$. 
Lemma 1.4. For any $n \geq 1$, all roots of $\mathcal{P}_{n}$ are located in $D_{1}$.

Proof. Since for $0 \leq m \leq n-1$ we have $0 \leq \frac{n^{m}}{m !} \leq \frac{n^{m+1}}{(m+1) !}$, the Eneström-Kakeya theorem [6] guarantees $\left|\zeta_{k}(n)\right| \leq 1(1 \leq k \leq n)$.

\section{Bounding $\mathcal{S}_{n}$ by a disk from outside}

In this section, we give an asymptotically optimal bound on the smallest disk containing $\mathcal{S}_{n}$, as well as uniform bounds valid for all $n$. First we prove the following inclusion for all positive $n$.

Lemma 2.1. We have $\mathcal{S}_{n} \subset D_{2}$ for any $n \geq 1$.

Proof. From the product representation $\mathcal{P}_{n}(z)=\frac{n^{n}}{n !} \prod_{k=1}^{n}\left(z-\zeta_{k}(n)\right)$ at $z=0$ we obtain $\frac{n^{n}}{n !}=\prod_{k=1}^{n} \frac{1}{-\zeta_{k}(n)}$. Hence for any $z \in \mathbb{C}$ we have $\left|\mathcal{P}_{n}(z)\right|=\frac{n^{n}}{n !} \prod_{k=1}^{n}\left|z-\zeta_{k}(n)\right|=\prod_{k=1}^{n} \frac{\left|z-\zeta_{k}(n)\right|}{\left|\zeta_{k}(n)\right|} \geq$ $\prod_{k=1}^{n}\left|z-\zeta_{k}(n)\right|$, by Lemma 1.4 . Therefore, if $z \in \mathbb{C}$ is chosen such that $|z|>2$, then, due to $\left|\zeta_{k}(n)\right| \leq 1$ again, $\left|z-\zeta_{k}(n)\right|>1$, so $\left|\mathcal{P}_{n}(z)\right|>\prod_{k=1}^{n} 1$, and thus $z \notin \mathcal{S}_{n}$.

Next we refine the above result for $n$ large enough.

Theorem 2.2. For each $\varepsilon \in(0,1)$ and $n \geq n_{0}(\varepsilon):=\left(\frac{1.0085 e}{\varepsilon}\right)^{2}$, we have $\mathcal{S}_{n} \subset D_{1+\varepsilon}$.

Proof. Let us fix $\varepsilon \in(0,1)$. Then for any $n \geq 1$ and $|z| \geq 1$ we have $\left|T_{n}(z)\right| \leq e \sqrt{n}$ by (5). So, due to the Cauchy inequality for the derivative, $\left|T_{n}^{\prime}(z)\right| \leq e \sqrt{n} / \varepsilon$ for $|z| \geq 1+\varepsilon$. Now for any $n>(e / \varepsilon)^{2}$ and $|z| \geq 1+\varepsilon$, we get $\left|T_{n}^{\prime}(z)\right| / n \leq e /(\varepsilon \sqrt{n})<1$, so by (3) and by (17) in Lemma 6.7 with $\sigma:=1$ we have for such $n$ and $z$ values that

$$
\begin{aligned}
& \left|T_{n}(z)\right| \geq\left(\inf \left\{\left|\frac{w}{w-1}\right|: w \in \mathbb{C},|w| \geq 1+\varepsilon\right\}\right)\left(1-\frac{\left|T_{n}^{\prime}(z)\right|}{n}\right) \geq \\
& \left(\inf \left\{\left|\frac{w}{w-1}\right|: w \in \mathbb{C},|w| \geq 1\right\}\right)\left(1-\frac{e}{\varepsilon \sqrt{n}}\right)=\frac{1}{2}\left(1-\frac{e}{\varepsilon \sqrt{n}}\right) .
\end{aligned}
$$


But $\frac{1}{2}\left(1-\frac{e}{\varepsilon \sqrt{n}}\right) \geq 17 / 4034$ for $n \geq n_{0}(\varepsilon)$, hence for any such $n$ and $|z| \geq 1+\varepsilon$ we obtain $\left|\mathcal{P}_{n}(z)\right|=\frac{n^{n}|z|^{n}}{n !}\left|T_{n}(z)\right| \geq 17 / 4034 \cdot \frac{n^{n}}{n !}$. Now, by Lemma 6.1. $\frac{n^{n}}{n !} \geq \frac{e^{n}}{e \sqrt{n}}$, and the function $[1,+\infty) \ni n \mapsto \frac{e^{n}}{e \sqrt{n}}$ is increasing. So for $\varepsilon \in(0,1), n \geq n_{0}(\varepsilon)$ and $|z| \geq 1+\varepsilon$ we obtain

$$
\left|\mathcal{P}_{n}(z)\right| \geq 17 / 4034 \cdot \frac{e^{n_{0}(\varepsilon)}}{e \sqrt{n_{0}(\varepsilon)}}=\frac{17000}{4068289} \cdot \varepsilon \cdot \frac{e^{n_{0}(\varepsilon)}}{e^{2}} \geq \frac{17000}{4068289} \cdot 1 \cdot \frac{e^{(1.0085 e / 1)^{2}}}{e^{2}}>1.03>1 .
$$

Remark 2.3. Theorem 2.2 is asymptotically optimal in the sense that the constant 1 in $D_{1+\varepsilon}$ cannot be replaced by a smaller positive number. This can be seen by noting that $1 \in \partial \Sigma_{1} \subset \mathcal{S}_{\infty}$ and using Theorem 1.3. On the other hand, at least for the $3 \leq n \leq 20$ values, we have $\mathcal{S}_{n} \subset D_{1}$. It would be interesting to investigate whether $\mathcal{S}_{n} \subset D_{1}$ holds for all $n \geq 21$ as well.

Finally, we use Theorem 2.2 to improve the bound in Lemma 2.1 for $n \geq 2$. In order to do so, we have determined the quantities $\max _{z \in \mathcal{S}_{n}}|z|$ as exact algebraic numbers for the first few $n$ values by transforming the problem from $\mathbb{C}$ to $\mathbb{R}^{2}$ and applying Mathematica's Maximize command with objective function $\sqrt{x^{2}+y^{2}}$. The resulting values are presented in Table 1. For the sake of brevity, instead of listing any exact algebraic numbers (in the $n=15$ case, for example, the algebraic degree of the exact maximum is 420 , while in the $n=20$ case, the degree is 760 ), their values are rounded up, so Table 1 provides strict upper bounds on $\max _{z \in \mathcal{S}_{n}}|z|$. These values can be computed quickly: even the $n=20$ case was obtained in just 40 seconds (on a laptop, as of 2013). We remark that the maximum is attained within $\{\Re<0\}$ for $1 \leq n \leq 4$, and within $\{\Re>0\}$ for $5 \leq n \leq 20$.

Corollary 2.4. For each $n \geq 2$, we have $\mathcal{S}_{n} \subset D_{1.6}$.

Proof. For $2 \leq n \leq 20$, the result follows from the computations given in Table 1. For $n \geq 21$, we can apply Theorem 2.2 with $\varepsilon:=0.6$ because $21>n_{0}(\varepsilon)>20.87$.

\section{Bounding $\mathcal{S}_{n}$ in the left half-plane by a semi-disk from outside}

Figures 2 and 4(b) suggest that the size of the bounding disks in Section 2 is dictated by the locations of zeros of $\mathcal{P}_{n}$ in the right half-plane. In numerical analysis, we are typically interested in stability behavior only in the left half-plane. For $n>4$, the portion of $\mathcal{S}_{n}$ in the left half-plane is apparently contained in a smaller disk. Theorem 3.1 and Corollary 3.2 give an asymptotic result, while Theorem 3.4 is valid for all $n \geq 3$. 


\begin{tabular}{c|c||c|c}
$n$ & $\max _{z \in \mathcal{S}_{n}}|z|$ & $n$ & $\max _{z \in \mathcal{S}_{n}}|z|$ \\
\hline 1 & 2 & 11 & 0.664 \\
2 & $\frac{1}{2} \sqrt{2(1+\sqrt{2})} \approx 1.099$ & 12 & 0.670 \\
3 & 0.847 & 13 & 0.676 \\
4 & 0.741 & 14 & 0.682 \\
5 & 0.690 & 15 & 0.687 \\
6 & 0.665 & 16 & 0.692 \\
7 & 0.6546 & 17 & 0.697 \\
8 & 0.6523 & 18 & 0.702 \\
9 & 0.6542 & 19 & 0.707 \\
10 & 0.659 & 20 & 0.711 \\
\hline
\end{tabular}

Table 1: For $n \geq 3$, the exact values of $\max _{z \in \mathcal{S}_{n}}|z|$ (rounded up). In the given range $1 \leq n \leq 20$, the minimum of $\max _{z \in \mathcal{S}_{n}}|z|$ is attained for $n=8$.

Theorem 3.1. For any $\varepsilon>0$ there exists $n_{0}(\varepsilon) \in \mathbb{N}^{+}$such that $n \geq n_{0}(\varepsilon)$ implies

$$
\mathcal{S}_{n} \cap\{\Re \leq 0\} \subset D_{1 / e+\varepsilon} \cap\{\Re \leq 0\} .
$$

Proof. Let us fix any $\varepsilon>0$, and set $\varrho_{\varepsilon}:=1 / e+\varepsilon$ and $\delta_{\varepsilon}:=\varepsilon e / \sqrt{e^{2}+1}$. Then for any $\varrho \geq \varrho_{\varepsilon}$ and $z \in C_{\varrho}$, we know by Lemma 6.5 that $\left|z, \Sigma_{1}\right| \geq \delta_{\varepsilon}$. On the other hand, (4) implies $\left|T_{n}(z)\right| \leq 2 e \sqrt{n}$ for $z \notin \Sigma_{1}$, then the Cauchy inequality for the derivative that $\left|T_{n}^{\prime}(z)\right| \leq 2 e \sqrt{n} / \delta_{\varepsilon}$ for $\left|z, \Sigma_{1}\right| \geq \delta_{\varepsilon}$. So we get that

$$
\exists \widetilde{n}(\varepsilon) \in \mathbb{N} \quad \forall n \geq \widetilde{n}(\varepsilon) \quad \forall \varrho \geq \varrho_{\varepsilon} \quad \forall z \in C_{\varrho}: \quad \frac{\left|T_{n}^{\prime}(z)\right|}{n} \leq \frac{2 e}{\delta_{\varepsilon} \sqrt{n}}<1 .
$$

Then for such $n$ and $z$ values we proceed as in (6): by $\varrho_{\varepsilon}>1 / e$ and (17) in Lemma 6.7 with $\sigma:=1 / e$ we have that

$$
\begin{gathered}
\left|T_{n}(z)\right| \geq\left(\inf \left\{\left|\frac{w}{w-1}\right|: w \in \mathbb{C},|w| \geq \varrho_{\varepsilon}, \Re(w) \leq 0\right\}\right)\left(1-\frac{\left|T_{n}^{\prime}(z)\right|}{n}\right) \geq \\
\left(\inf \left\{\left|\frac{w}{w-1}\right|: w \in \mathbb{C},|w| \geq \frac{1}{e}\right\}\right)\left(1-\frac{2 e}{\delta_{\varepsilon} \sqrt{\widetilde{n}(\varepsilon)}}\right)=\frac{1}{1+e}\left(1-\frac{2 e}{\delta_{\varepsilon} \sqrt{\widetilde{n}(\varepsilon)}}\right)>0 .
\end{gathered}
$$

This yields, again by Lemma 6.1, for $n \geq \widetilde{n}(\varepsilon), \varrho \geq \varrho_{\varepsilon}$ and $z \in C_{\varrho}$ that

$$
\left|\mathcal{P}_{n}(z)\right|=\frac{n^{n}|z|^{n}}{n !}\left|T_{n}(z)\right| \geq \frac{|z|^{n} e^{n}}{(1+e) e \sqrt{n}}\left(1-\frac{2 e}{\delta_{\varepsilon} \sqrt{\widetilde{n}(\varepsilon)}}\right) \geq \frac{(1+\varepsilon e)^{n}}{(1+e) e \sqrt{n}}\left(1-\frac{2 e}{\delta_{\varepsilon} \sqrt{\widetilde{n}(\varepsilon)}}\right),
$$


and the last right-hand side is $>1$ for all $n$ larger than a suitable $n_{0}(\varepsilon)$.

By repeating the above proof with natural modifications, we obtain the following (more effective) version.

Corollary 3.2. Let us fix any $\delta>0$ and set $\varrho_{n}:=\frac{1}{e}+\frac{(2+\delta) \sqrt{e^{2}+1}}{\sqrt{n}}$. Then for any $n \in \mathbb{N}^{+}$we have

$$
\mathcal{S}_{n} \cap\{\Re \leq 0\} \subset D_{\varrho_{n}} \cap\{\Re \leq 0\} .
$$

Remark 3.3. Like Theorem 2.2. Theorem 3.1 is asymptotically optimal: the constant 1/e in $D_{1 / e+\varepsilon}$ cannot be replaced by a smaller positive number, since $-1 / e \in \mathcal{S}_{\infty}$ (cf. Remark 2.3).

Table 2 contains the values $\max \left\{|z|: z \in \mathcal{S}_{n} \cap\{\Re \leq 0\}\right\}$ for $1 \leq n \leq 20$. It can be seen from Figures 1 and 2 that, for larger $n$ values, the set $\mathcal{S}_{n} \cap\{\Re \leq 0\}$ is close to a semi-disk centered at the origin; see also the radial slices at the end of Section 5 . For $1 \leq n \leq 20$, the set $\mathcal{S}_{n} \cap\{\Re \leq 0\}$ can be covered by a disk with radius slightly less than $\frac{1}{e}+\frac{\ln n}{2 e n}+\frac{1.64}{n} ; c f$. Theorems 1.3 and 3.1, Remark 3.6, and observation $\mathrm{O}_{3}$ in the beginning of Section 4. Also notice that the asymptotic series of $\sqrt[n]{\frac{n !}{n^{n}}}($ as $n \rightarrow+\infty)$ starts with $\frac{1}{e}+\frac{\ln n}{2 e n}+\frac{\ln (2 \pi)}{2 e n}$.

\begin{tabular}{c|c||c|c}
$n$ & $\max _{z \in \mathcal{S}_{n} \cap\{\Re \leq 0\}}|z|$ & $n$ & $\max _{z \in \mathcal{S}_{n} \cap\{\Re \leq 0\}}|z|$ \\
\hline 1 & 2 & 11 & 0.496 \\
2 & $\frac{1}{2} \sqrt{2(1+\sqrt{2})} \approx 1.099$ & 12 & 0.486 \\
3 & 0.847 & 13 & 0.480 \\
4 & 0.741 & 14 & 0.476 \\
5 & 0.680 & 15 & 0.474 \\
6 & 0.597 & 16 & 0.458 \\
7 & 0.566 & 17 & 0.453 \\
8 & 0.546 & 18 & 0.450 \\
9 & 0.534 & 19 & 0.449 \\
10 & 0.527 & 20 & 0.448 \\
\hline
\end{tabular}

Table 2: For $n \geq 3$, the exact values of $\max _{z \in \mathcal{S}_{n} \cap\{\Re \leq 0\}}|z|$ (rounded up).

Theorem 3.4. For each $n \geq 3$ we have

$$
\mathcal{S}_{n} \cap\{\Re \leq 0\} \subset D_{0.95} \cap\{\Re \leq 0\} .
$$


Proof. The computations in Table 2 prove the statement of the theorem for $3 \leq n \leq 20$. So, due to Lemma 2.1 also, it is enough to show that $\left|\mathcal{P}_{n}(z)\right|>1$ for any $z \in C_{\varrho}$ with $\varrho \in[0.95,2]$ and $n \geq 21$.

Let us set $\delta_{\varrho}:=(\varrho e-1) /\left(2 \sqrt{e^{2}+1}\right)>0$. Then (4) implies $\left|T_{n}(z)\right| \leq 2 e \sqrt{n}$ for $z \notin \Sigma_{1}$, and the Cauchy inequality for the derivative that $\left|T_{n}^{\prime}(z)\right| \leq 2 e \sqrt{n} / \delta_{\varrho}$ for $\left|z, \Sigma_{1}\right| \geq \delta_{\varrho}$ and, say, $\Re(z) \leq \delta_{\varrho}$. Now from (3) we obtain

$$
\left|T_{n}(z)\right| \leq\left(\sup \left\{\left|\frac{w}{w-1}\right|: w \in \mathbb{C},\left|w, \Sigma_{1}\right| \geq \delta_{\varrho}, \Re(w) \leq \delta_{\varrho}\right\}\right)\left(1+\frac{2 e}{\delta_{\varrho} \sqrt{n}}\right)
$$

for $z$ with $\left|z, \Sigma_{1}\right| \geq \delta_{\varrho}, \Re(z) \leq \delta_{\varrho}$. We notice that for any $z$ with $\left|z, \Sigma_{1}\right| \geq 2 \delta_{\varrho}, \Re(z) \leq 0$ we have

$$
D_{\delta_{\varrho}}(z) \subset\left\{w \in \mathbb{C}:\left|w, \Sigma_{1}\right| \geq \delta_{\varrho}, \Re(w) \leq \delta_{\varrho}\right\},
$$

so (7) and the Cauchy inequality for the derivative again yield

$$
\frac{\left|T_{n}^{\prime}(z)\right|}{n} \leq \frac{1}{n \delta_{\varrho}}\left(\sup \left\{\left|\frac{w}{w-1}\right|: w \in \mathbb{C},\left|w, \Sigma_{1}\right| \geq \delta_{\varrho}, \Re(w) \leq \delta_{\varrho}\right\}\right)\left(1+\frac{2 e}{\delta_{\varrho} \sqrt{n}}\right)
$$

for any $z$ with $\left|z, \Sigma_{1}\right| \geq 2 \delta_{\varrho}, \Re(z) \leq 0$. We check with the help of Lemma 6.7 that the right-hand side of $(8)$ is $<1$ for all $n \geq 21$ and $\varrho \in\left[0.95, \frac{1+\sqrt{1+e^{2}}}{e}\right] \cup\left(\frac{1+\sqrt{1+e^{2}}}{e}, 2\right]$.

Now by Lemma 6.4 we see that for any $w \in \mathbb{C}$ and $\left|w, \Sigma_{1}\right| \geq 2 \delta_{\varrho}$ we have $|w| \geq 1 / 4$. So by (17) in Lemma 6.7 with $\sigma:=1 / 4$ and by (3) again we showed that for $n \geq 21,\left|z, \Sigma_{1}\right| \geq 2 \delta_{\varrho}$ and $\Re(z) \leq 0$ we have

$$
\begin{gathered}
\left|T_{n}(z)\right| \geq\left(\inf \left\{\left|\frac{w}{w-1}\right|: w \in \mathbb{C},\left|w, \Sigma_{1}\right| \geq 2 \delta_{\varrho}, \Re(w) \leq 0\right\}\right)\left(1-\frac{\left|T_{n}^{\prime}(z)\right|}{n}\right) \geq \\
\left(\inf \left\{\left|\frac{w}{w-1}\right|: w \in \mathbb{C},|w| \geq 1 / 4\right\}\right)\left(1-\frac{\left|T_{n}^{\prime}(z)\right|}{n}\right)=\frac{1}{5}\left(1-\frac{\left|T_{n}^{\prime}(z)\right|}{n}\right) \geq \\
\frac{1}{5}\left(1-\frac{1}{n \delta_{\varrho}}\left(\sup \left\{\left|\frac{w}{w-1}\right|: w \in \mathbb{C},\left|w, \Sigma_{1}\right| \geq \delta_{\varrho}, \Re(w) \leq \delta_{\varrho}\right\}\right)\left(1+\frac{2 e}{\delta_{\varrho} \sqrt{n}}\right)\right) .
\end{gathered}
$$

We use Lemma 6.7 one more time to verify that this last lower estimate of $\left|T_{n}(z)\right|$ is $\geq 4 \cdot 10^{-4}$ for $n \geq 21$ and $\varrho \in\left[0.95, \frac{1+\sqrt{1+e^{2}}}{e}\right] \cup\left(\frac{1+\sqrt{1+e^{2}}}{e}, 2\right]$.

Finally, from Lemma 6.5 and the definition of $\delta_{\varrho}$ we see that $\varrho \in[0.95,2], z \in C_{\varrho}$ and $n \geq 21$ imply $\left|z, \Sigma_{1}\right| \geq 2 \delta_{\varrho}$ and $\Re(z) \leq 0$, and hence

$$
\left|T_{n}(z)\right| \geq 4 \cdot 10^{-4}
$$


Therefore, for $\varrho \in[0.95,2], z \in C_{\varrho}$ and $n \geq 21$ (by Lemma 6.1 also) we obtain

$$
\left|\mathcal{P}_{n}(z)\right|=\frac{n^{n}|z|^{n}}{n !}\left|T_{n}(z)\right| \geq \frac{n^{n} \cdot 0.95^{n}}{n !} \cdot 4 \cdot 10^{-4} \geq \frac{e^{n} \cdot 0.95^{n}}{e \sqrt{n}} \cdot 4 \cdot 10^{-4}>14422>1 .
$$

Remark 3.5. One could slightly reduce the constant 0.95 in $D_{0.95}$ by performing another iteration (or several more iterations) in the proof above (an estimate on $T \rightsquigarrow$ an estimate on $T^{\prime} \rightsquigarrow$ an estimate on $T$, and so on), but then the starting index 21 in $n \geq 21$ would jump to a much higher value.

Remark 3.6. The main obstacle to improve the bounds appearing in Theorems 2.2 and 3.4 is that we could only guarantee the positivity of $\left|1+\frac{T_{n}^{\prime}(z)}{n}\right|$ by applying the inequality $|a-b| \geq|| a|-| b||$ and choosing large $n$ values. For example, in order for Corollary 3.2 to be stronger than the estimate in Corollary 2.4, we need $n \geq 23$, and to be stronger than the estimate in Theorem 3.4, we need $n \geq 100$. The convergence rate $\mathcal{O}\left(\frac{1}{\sqrt{n}}\right)$ presented in Corollary 3.2 naturally follows from the estimates on $\left|T_{n}\right|$ and $\left|T_{n}^{\prime}\right|$; we do not know whether this rate can be improved. As a related result, [4] proves that the convergence rate of the zeros $\zeta_{k}(n)$ in (1) to $\partial \Sigma_{1}$ is exactly $\mathcal{O}\left(\frac{1}{\sqrt{n}}\right)$ as $n \rightarrow+\infty$, but the rate improves to $\mathcal{O}\left(\frac{\ln n}{n}\right)$ if zeros in a small disk $D_{\varepsilon}(1)(\varepsilon \in(0,1]$ arbitrary but fixed) are ignored.

\section{The stability region near the imaginary axis}

In this section we are concerned with the imaginary part of the "upper vertical slice" of $\mathcal{U}_{n}$ along the imaginary axis,

$$
\mathcal{V}_{n}^{+}:=\left\{\Im(z): z \in \mathbb{C}, \Re(z)=0, \Im(z) \geq 0,\left|\sum_{k=0}^{n} \frac{z^{k}}{k !}\right| \leq 1\right\} \quad\left(n \in \mathbb{N}^{+}\right) .
$$

We focus only on the upper half-plane due to symmetry, and use the unscaled regions because they lead to simpler expressions. By also taking into account the explicit representations given in Lemma 4.1 below, it is easy to determine the set $\mathcal{V}_{n}^{+}$for a particular $n$; see Figure 6 for $1 \leq n \leq 20$, and Figure 7 for $1 \leq n \leq 100$. Based on these figures and on the exact represenation of the endpoints of the shaded intervals as Root objects in Mathematica, the following observations are made.

$\mathrm{O}_{1}$. For $n \equiv 0 \bmod 4$ or $n \equiv 3 \bmod 4$, the connected component of $\mathcal{V}_{n}^{+}$containing the origin is an interval of positive length, while for $n \equiv 1 \bmod 4$ or $n \equiv 2 \bmod 4$ the corresponding interval is the singleton $\{0\}$. 
$\mathbf{O}_{2}$. For $n \geq 1, \mathcal{V}_{n}^{+}$consists of disjoint compact intervals whose endpoints tend to the grid $\{\pi \ell: \ell \in \mathbb{N}\}$ as $n \rightarrow+\infty$ for even $n$, or to the grid $\{0\} \cup\{\pi / 2+\pi \ell: \ell \in \mathbb{N}\}$ as $n \rightarrow+\infty$ for odd $n$. For a fixed and large enough $n$, non-degenerate intervals in $\mathcal{V}_{n}^{+}$or in the complement of $\mathcal{V}_{n}^{+}$alternate one after another as we move away from the origin; the pattern starts according to the rule described by $\mathbf{O}_{1}$.

$\mathrm{O}_{3}$. The farthest point of $\mathcal{V}_{n}^{+}$from the origin is bounded from above by $\frac{n}{e}+\frac{\ln n}{2 e}+1.2604$ for $1 \leq n \leq 100$ (see the top red curve in Figure 7 ).

In the following we explain observations $\mathbf{O}_{1}$ and $\mathbf{O}_{2}$, and indicate how $\mathbf{O}_{3}$ is related to some earlier results in the literature. At the end of the section we also investigate the boundary curve of $\mathcal{U}_{n}$ that oscillates around the imaginary axis: intersection points of the boundary curve and the upper semi-axis define the endpoints of the shaded intervals in the corresponding column of Figure 7 - we illustrate the amplitude of these oscillations for different $n$ values.

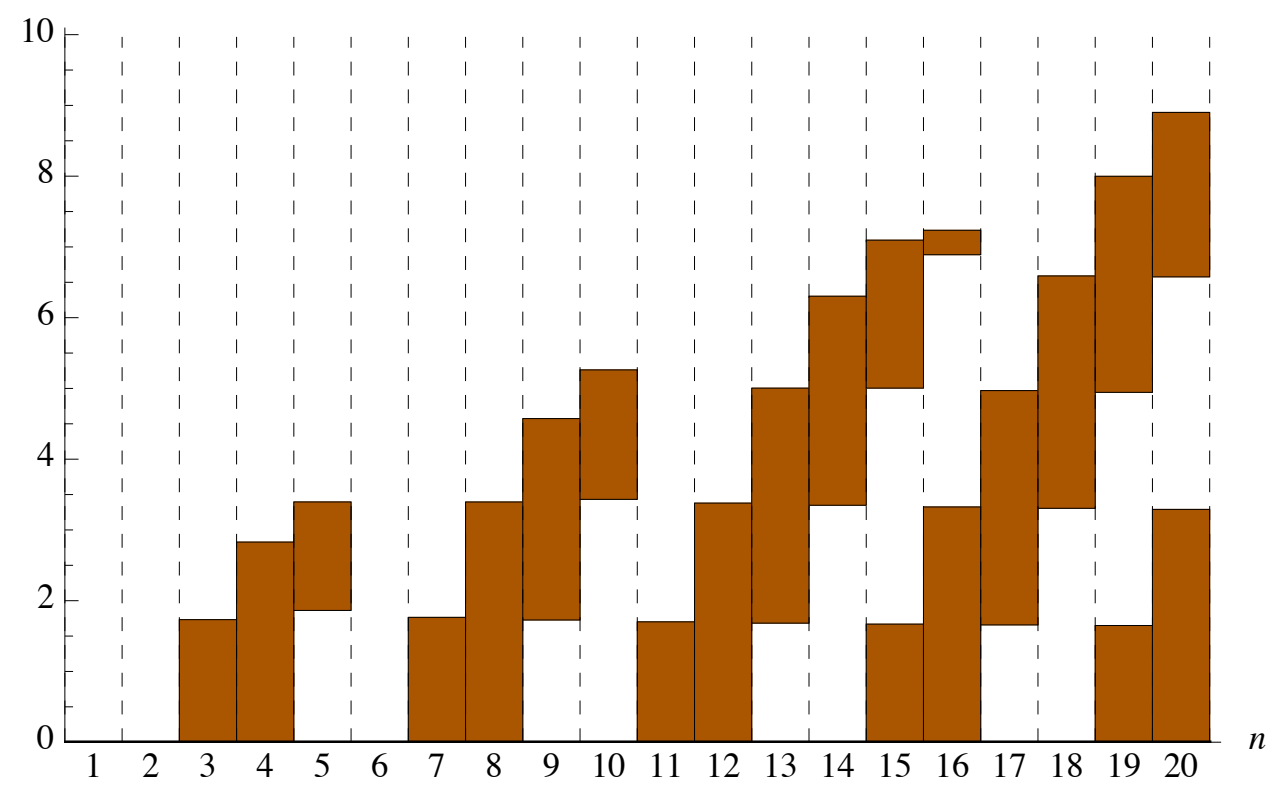

Figure 6: For each $1 \leq n \leq 20$, the intervals constituting $\mathcal{V}_{n}^{+}$are represented as shaded rectangles.

The first lemma explicitly describes the absolute value of the $n^{\text {th }}$ Taylor polynomial of the exponential function along the imaginary axis, simultaneously providing us with "finite truncations" of the identity $\cos ^{2} y+\sin ^{2} y=1$. It is convenient to refer to the $E$-polynomial, 


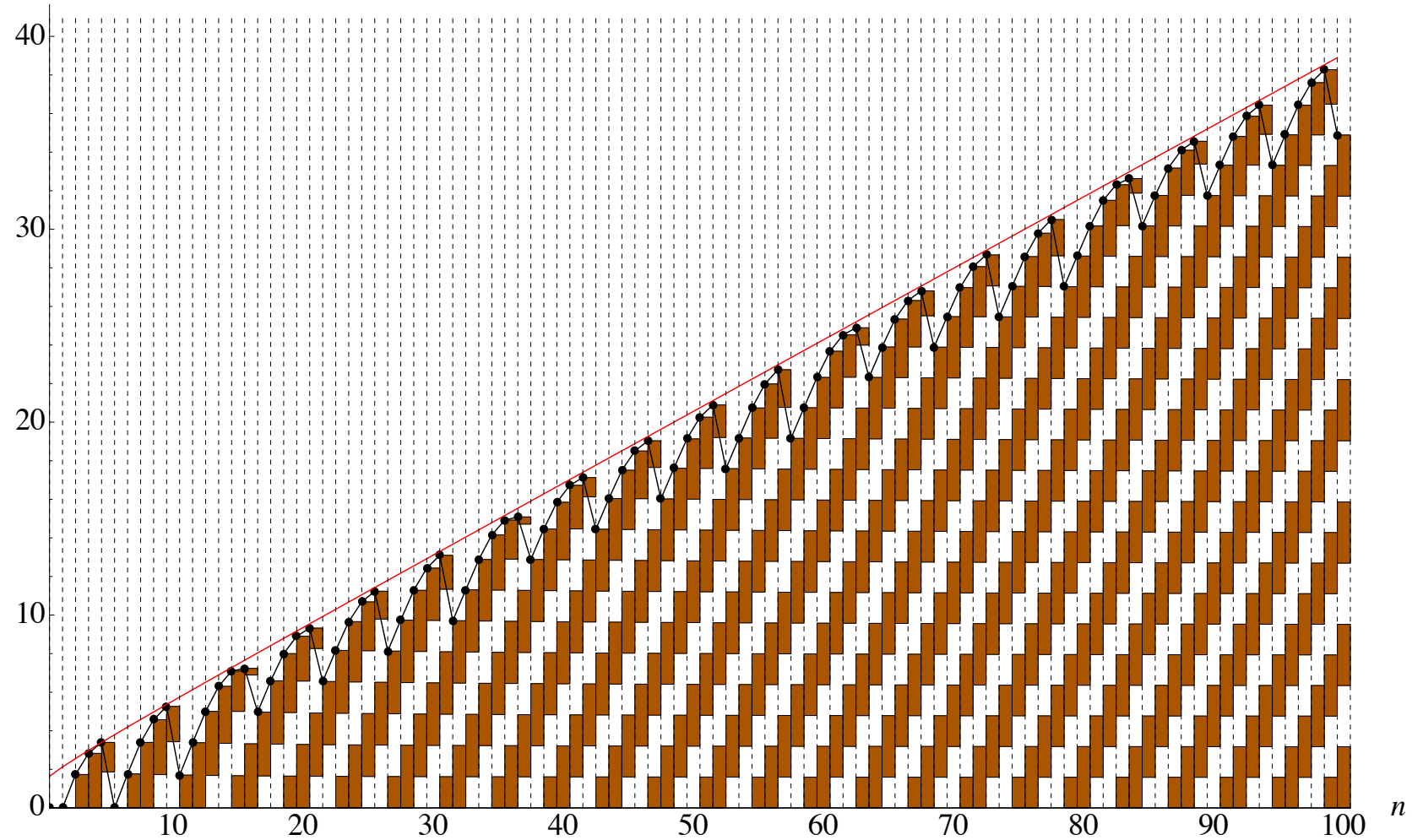

Figure 7: Extension of Figure 6 for $1 \leq n \leq 100$. Black dots (with piecewise linear interpolation) show the sequence $\max \left(\mathcal{V}_{n}^{+}\right)$. The blocks consisting of monotone non-decreasing adjacent elements have length $5,5,6,5,5,5,6,5,5,5,5,6,5,5,5,6,5,5,5$ in the given range ( $c f$. the "separation of bubbles" in Figure 1). The top red curve, being the graph of $n \mapsto \frac{n}{e}+\frac{\ln n}{2 e}+1.2604$, is an upper estimate in the given range.

which was used in [26] to study the behavior of rational functions along the imaginary axis. For the polynomials studied here, the $E$-polynomial takes the form

$$
E_{n}(y):=\left|\sum_{k=0}^{n} \frac{(i y)^{k}}{k !}\right|^{2}-1 \quad\left(y \in \mathbb{R}, n \in \mathbb{N}^{+}\right) .
$$


Lemma 4.1. For any $y \in \mathbb{R}$ and $n \geq 1$ integer we have

$$
E_{n}(y)=\left\{\begin{array}{lll}
-\frac{y^{n+1}}{n !} \sum_{k=1}^{n / 2} \frac{(-1)^{k+1} y^{2 k-1}}{(2 k-1) !\left(k+\frac{n}{2}\right)}, & \text { if } n \equiv 0 & \bmod 4 \\
\frac{y^{n+1}}{n !} \sum_{k=0}^{\frac{n-1}{2}} \frac{(-1)^{k} y^{2 k}}{(2 k) !\left(k+\frac{n+1}{2}\right)}, & \text { if } n \equiv 1 & \bmod 4 \\
\frac{y^{n+1}}{n !} \sum_{k=1}^{n / 2} \frac{(-1)^{k+1} y^{2 k-1}}{(2 k-1) !\left(k+\frac{n}{2}\right)}, & \text { if } n \equiv 2 & \bmod 4 \\
-\frac{y^{n+1}}{n !} \sum_{k=0}^{\frac{n-1}{2}} \frac{(-1)^{k} y^{2 k}}{(2 k) !\left(k+\frac{n+1}{2}\right)}, & \text { if } n \equiv 3 & \bmod 4 .
\end{array}\right.
$$

The straightforward proof of the above lemma is omitted. The following corollary shows that the sign of the lowest order term of $E_{n}$ explains $\mathbf{O}_{1}$.

Corollary 4.2. For any positive integer $n$ with $n \equiv 1 \bmod 4$ or $n \equiv 2 \bmod 4$, there exists $\varrho_{n}>0$ such that

$$
\mathcal{V}_{n}^{+} \cap\left[0, \varrho_{n}\right]=\{0\}
$$

On the other hand, for any positive integer $n$ with $n \equiv 0 \bmod 4$ or $n \equiv 3 \bmod 4$, there exists $\varrho_{n}>0$ such that

$$
\left[0, \varrho_{n}\right] \subset \mathcal{V}_{n}^{+} \text {. }
$$

Proof. By Lemma 4.1 we have for $n \equiv 0 \bmod 4$ and $y \in \mathbb{R}$ that

$$
E_{n}(y)=-\frac{1}{\left(1+\frac{n}{2}\right) n !} y^{n+2}+\mathcal{O}\left(y^{n+4}\right)
$$

as $y \rightarrow 0$, so for some $\varrho_{n}>0$ sufficiently small, $E_{n}(y) \leq 0$ for all $y \in\left[0, \varrho_{n}\right]$. But

$$
\mathcal{V}_{n}^{+}=\left\{y \in \mathbb{R}: y \geq 0, E_{n}(y) \leq 0\right\} \quad\left(n \in \mathbb{N}^{+}\right),
$$

therefore this case is finished. The proof for the other three congruence classes is analogous.

We begin explaining $\mathbf{O}_{2}$ by noting that for each $n \geq 1, \lim _{y \rightarrow+\infty} E_{n}(y)=+\infty$ and $E_{n}(0)=0$, so there exist an index $k_{0}(n) \in \mathbb{N}^{+}$and some mutually disjoint non-empty compact intervals $I_{n, k}\left(k=1,2, \ldots, k_{0}(n)\right)$ such that

$$
\mathcal{V}_{n}^{+}=\left\{y \in \mathbb{R}: y \geq 0, E_{n}(y) \leq 0\right\}=\bigcup_{k=1}^{k_{0}(n)} I_{n, k} .
$$


These $I_{n, k}$ intervals are just the shaded vertical rectangles in Figure 6 or 7 . It of course can happen that $k_{0}(n)=1$, or some of the $I_{n, k}$ intervals are singletons. We order the intervals $I_{n, k}$ in the natural way so that $\min I_{n, 1}=0$ and $\max I_{n, k}<\min I_{n, k+1}\left(k=1,2, \ldots, k_{0}(n)-1\right)$. Let us consider now the scaled counterpart of the polynomial $E_{n}$ :

$$
\widetilde{E}_{n}(y):=\frac{(n+1) !}{2 y^{n+1}} E_{n}(y) \quad(y \in \mathbb{R} \backslash\{0\}) .
$$

Then $\mathcal{V}_{n}^{+}=\{0\} \cup\left\{y \in \mathbb{R}: y>0, \widetilde{E}_{n}(y) \leq 0\right\}$. The advantage of $\widetilde{E}_{n}$ over $E_{n}$ is that for

$$
n \equiv 0, n \equiv 1, n \equiv 2 \text { and } n \equiv 3 \quad \bmod 4 \text {, }
$$

$\widetilde{E}_{n}(y)$ is a perturbation of the truncated series for

$$
-\sin y \equiv-\sum_{k=1}^{\infty} \frac{(-1)^{k+1} y^{2 k-1}}{(2 k-1) !}, \cos y, \sin y \text { and }-\cos y,
$$

respectively; see Figure 8. This motivates us to use some tools from complex analysis to study the positive real roots of $\widetilde{E}_{n}$. In what follows, we again focus only on the $n \equiv 0 \bmod 4$ case, because the explanation of $\mathbf{O}_{2}$ for the other three congruence classes is analogous.

Let $f_{m}$ denote the extension of $\widetilde{E}_{n}$ with $n=4 m$ to all of $\mathbb{C}$ :

$$
f_{m}(z)=-\frac{(4 m+1)}{2} \sum_{k=1}^{2 m} \frac{(-1)^{k+1} z^{2 k-1}}{(2 k-1) !(k+2 m)} \quad\left(z \in \mathbb{C}, m \in \mathbb{N}^{+}\right)
$$

Clearly,

$$
\mathcal{V}_{4 m}^{+}=\left\{y \in \mathbb{R}: y \geq 0, f_{m}(y) \leq 0\right\} .
$$

The following lemma makes the word "perturbation" above more precise.

Lemma 4.3. The sequence of polynomials $f_{m}$ defined by 10 converges uniformly to the function - sin on compact subsets of $\mathbb{C}$ as $m \rightarrow+\infty$.

Proof. Let us fix some $\varrho>0$. We know that the sequence of Maclaurin polynomials $\widetilde{f}_{m}(z):=$ $-\sum_{k=0}^{2 m-1} \frac{(-1)^{k} z^{2 k+1}}{(2 k+1) !}$ converges uniformly to $-\sin$ on $D_{\varrho}$ as $m \rightarrow+\infty$, so, by using the triangle inequality, it is enough to show that

$$
\lim _{m \rightarrow+\infty} \sup \left\{\left|f_{m}(z)-\widetilde{f}_{m}(z)\right|: z \in D_{\varrho}\right\}=0
$$




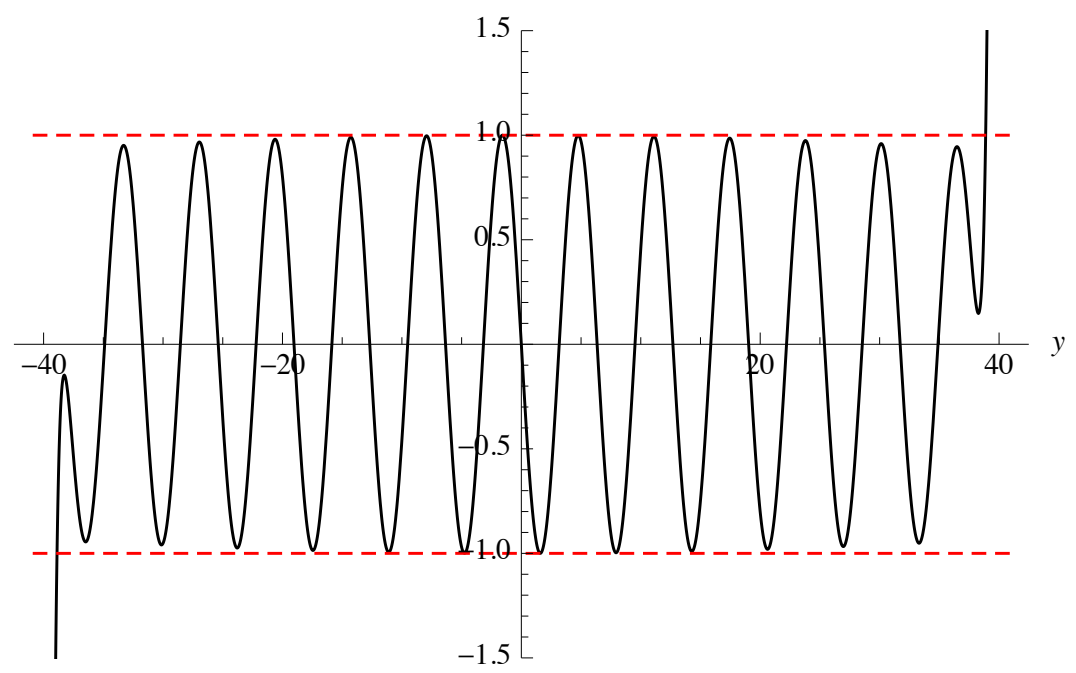

Figure 8: The graph of $\widetilde{E}_{100}$ over the interval $\left[-\frac{n}{e}-4, \frac{n}{e}+4\right]$.

But

$$
\begin{gathered}
\left|f_{m}(z)-\widetilde{f}_{m}(z)\right|=\left|\sum_{k=0}^{2 m-1} \frac{(-1)^{k} z^{2 k+1}}{(2 k+1) !} \cdot \frac{2 k+1}{2 k+2+4 m}\right| \leq \\
|z| \sum_{k=0}^{2 m-1} \frac{|z|^{2 k}}{(2 k) !} \cdot \frac{1}{2 k+2+4 m} \leq \frac{\varrho}{4 m} \sum_{k=0}^{\infty} \frac{\varrho^{2 k}}{(2 k) !}=\frac{\varrho \cosh \varrho}{4 m} \rightarrow 0,
\end{gathered}
$$

as $m \rightarrow+\infty$.

Remark 4.4. Notice that the first inequality in (12) holds with equality for purely imaginary $z$ values, and $\varrho \cosh \varrho$ is large when $\varrho$ is large. These already suggest - together with numerical computations - that the uniform convergence of $f_{m}$ to - sin on $D_{\varrho}$ is rather slow, compared to the uniform convergence of $\widetilde{f}_{m}$ to $-\sin$.

The uniform convergence of $f_{m}$ to - sin on compact subsets of $\mathbb{C}$ implies that the roots of $f_{m}$ converge to the roots of - sin. Let us elaborate on this in the following theorem to finish our explanation of $\mathrm{O}_{2}$ in the $n=4 \mathrm{~m}$ case. The theorem expresses the fact that for any fixed $k^{*} \in \mathbb{N}^{+}$there exists an index $m_{0}\left(k^{*}\right)$ such that for each $m>m_{0}\left(k^{*}\right)$ we have $k_{0}(4 m) \geq k^{*}$ in decomposition $(9)$, and the sequence of intervals $I_{4 m, k^{*}} \subset \mathcal{V}_{4 m}^{+}$converges to the interval $\left[\left(2 k^{*}-2\right) \pi,\left(2 k^{*}-1\right) \pi\right]$ as $m \rightarrow+\infty$. We essentially repeat the proof of Hurwitz's theorem via Rouché's theorem as given in [18, p. 119] ( $c f$. [21, pp. 374-375]). 
Theorem 4.5. For any fixed $k^{*} \geq 1$,

$$
\lim _{m \rightarrow+\infty} \min I_{4 m, k^{*}}=\left(2 k^{*}-2\right) \pi \quad \text { and } \quad \lim _{m \rightarrow+\infty} \max I_{4 m, k^{*}}=\left(2 k^{*}-1\right) \pi .
$$

Proof. We set $\varrho:=\left(2 k^{*}-1\right) \pi+\pi / 3, \varepsilon:=\pi / 4$ and let $\eta \equiv \eta(\varrho, \varepsilon):=\min \{|-\sin (z)|: z \in$ $\left.\partial D_{\varrho} \cup \partial D_{\varepsilon}\right\}$. Then for any $\ell \in \mathbb{Z}, 0<\eta \leq \min \left\{|-\sin (z)|: z \in \partial D_{\varepsilon}(\ell \pi)\right\}$. By Lemma 4.3 , there is an index $m_{0}(\varrho, \varepsilon)>0$ such that for all $m>m_{0}(\varrho, \varepsilon)$ we have

$$
\left|f_{m}-(-\sin )\right|<\eta \leq|-\sin | \quad \text { on } \quad \partial D_{\varrho} \cup \bigcup_{\ell=-2 k^{*}+1}^{2 k^{*}-1} \partial D_{\varepsilon}(\ell \pi) .
$$

So let us fix $m>m_{0}(\varrho, \varepsilon)$ arbitrarily. We show that the decomposition $(9)$ of $\mathcal{V}_{4 m}^{+}$consists of at least $k^{*}$ intervals - that is, $k_{0}(4 m) \geq k^{*}$-and $\max I_{4 m, k^{*}}<\varrho$.

Indeed, Rouché's theorem asserts, on one hand, that both $-\sin$ and $f_{m}$ have the same number of zeros (counted with multiplicity) in $D_{\varrho}$, that is, the number of zeros is $4 k^{*}-1$ for both functions; on the other hand, for each $\ell \in\left[-2 k^{*}+1,2 k^{*}-1\right] \cap \mathbb{Z}$ they have the same number of zeros in $D_{\varepsilon}(\ell \pi)$ as well, that is, they both have a unique zero in each such disk. Since $f_{m}$ has real coefficients, this means that $f_{m}$ has a unique real zero in $D_{\varepsilon}(\ell \pi)$ for each $\ell \in\left[-2 k^{*}+1,2 k^{*}-1\right] \cap \mathbb{Z}$, also implying that all complex zeros of $f_{m}$ in $D_{\varrho}$ are real and simple. But $f_{m}(0)=0$, so the number of zeros of $f_{m}$ in the interval $(0, \varrho)$ is exactly $2 k^{*}-1$. Let $0<y_{4 m, 1}<y_{4 m, 2}<\ldots<y_{4 m, 2 k^{*}-1}<\varrho$ denote these zeros of $f_{m}$, and set $y_{4 m, 0}:=0$. By the fact that zeros of $f_{m}$ are simple, and by taking into account Corollary 4.2 and (11), we have that $f_{m}(y) \leq 0$ for $y \in\left[y_{4 m, 0}, y_{4 m, 1}\right]$, and hence $f_{m}(y) \leq 0$ for $y \in \bigcup_{k=0}^{k^{*}-1}\left[y_{4 m, 2 k}, y_{4 m, 2 k+1}\right]$, and $f_{m}(y)>0$ for $y \in\left(\bigcup_{k=1}^{k^{*}-1}\left(y_{4 m, 2 k-1}, y_{4 m, 2 k}\right)\right) \cup\left(y_{4 m, 2 k^{*}-1}, \varrho\right]$. In other words, $\mathcal{V}_{4 m}^{+} \cap[0, \varrho]=\bigcup_{k=0}^{k^{*}-1}\left[y_{4 m, 2 k}, y_{4 m, 2 k+1}\right]$.

Finally, by repeating the above argument with some $\varepsilon_{j} \rightarrow 0^{+}\left(\varepsilon_{j} \leq \varepsilon, j=1,2, \ldots\right)$ instead of $\varepsilon$, the convergence of the endpoints of the $I_{4 m, k^{*}}$ intervals to the corresponding multiples of $\pi$ as $m \rightarrow+\infty$ is also established.

Remark 4.6. The convergence of the endpoints of the intervals $I_{4 m, k^{*}}\left(\right.$ as $m \rightarrow+\infty$ and $k^{*}$ is fixed) to the corresponding multiples of $\pi$ seems to be monotone if the first few $\left(I_{4, k^{*}}, I_{8, k^{*}}, \ldots\right)$ intervals are ignored, but we did not prove this. Moreover, Remark 4.4 indicates why this convergence is relatively slow.

Remark 4.7. It is interesting to apply the notions of order stars [26] to the current setting. By [26. Proposition 3], we know that near the origin, the order star for $\mathcal{P}_{n}$ and its complement each consist of $n+1$ alternating sectors of equal angular size. This leads easily to a proof of Corollary 4.2. Meanwhile, [26, Propositions 2 and 4] indicate that the order star for $\mathcal{P}_{n}$ has 
$n$ bounded dual fingers, each containing one zero of $\mathcal{P}_{n}$. The dual fingers correspond (near the origin) to the sectors belonging to the complement of the order star, so approximately half of them start in the right half-plane. But according to Szegö, more than half of the zeros of $\mathcal{P}_{n}$ lie in the left half plane. This means that a certain fraction of the dual fingers must cross the imaginary axis. These crossings correspond to the gaps in $\mathcal{V}_{n}^{+}$. If one supposes that each crossing and each gap between crossings have equal length, one obtains that the width of each finger where it crosses the imaginary axis must be $\pi$.

As for $\mathrm{O}_{3}$, we notice that the upper bound $\frac{n}{e}+\frac{\ln n}{2 e}+1.2604$ (or its possibly modified version for $n>100$ ) is an upper bound on the largest positive root of $\widetilde{E}_{n}$. Since these polynomials are uniformly close to $\pm \sin$ or $\pm \cos$ on compact sets of $\mathbb{C}$ for large $n$ values (Lemma 4.3), it is reasonable to expect (but we do not investigate this further here either) that the analysis presented in [21] is applicable in the current situation as well, at least for $n$ large enough: compare our Figure 9 with [21, Figures 1.1 and 1.2], or our expression $\frac{n}{e}+\frac{\ln n}{2 e}+1.2604$ with Szegö's asymptotic result [21, formula (1.12)] and its improvement [21, Section 5].

Let us close the section by presenting some further observations based on computations. In the numerical integration of oscillatory problems, one is interested in the size of the component of $\mathcal{V}_{n}^{+}$that is connected to the origin. The largest connected component of $\mathcal{V}_{n}^{+}(1 \leq n \leq 100)$ containing 0 occurs for $n=8$, when $\mathcal{V}_{8}^{+}=\left[0, y_{8,1}\right]$ with $y_{8,1} \approx 3.3951402205749$; the largest scaled component (i.e., when the $n^{\text {th }}$ column of Figure 7 is shrunk by a factor $n$ ) occurs for $n=4$. For large $n$, the connected component of $\mathcal{V}_{n}^{+}(1 \leq n \leq 100)$ containing 0 remains small, but the boundary of $\mathcal{U}_{n}$ lies very close to the imaginary axis and the magnitude of the amplification factor along the imaginary axis is only slightly greater than unity (indeed, indistinguishable in double precision) over a relatively large interval. We can exactly determine the distance from the boundary of $\mathcal{U}_{n}$ to the imaginary axis for a given $n$ with Mathematica: as an illustration, we chose $n=6$ (Figure $10(\mathrm{a}), n=20$ (Figure 10(b)) and $n=100$ (Figure 11). Below we describe the technique we used to create these figures.

For a given $y \geq 0$, the real solutions $x$ of $\left|\sum_{k=0}^{n} \frac{(x+i y)^{k}}{k !}\right| \leq 1$ are computed and the solution with the smallest absolute value is denoted by $x_{n}^{\min }(y)$. In Mathematica, the function $y \mapsto x_{n}^{\min }(y)$ (where $y$ runs over some interval) can be represented as a piecewise defined function composed of Root objects. However, the $x_{n}^{\min }(\cdot)$ function typically spans several orders of magnitude, for example,

$$
\begin{gathered}
x_{100}^{\min }(0.1) \approx 10^{-262}, x_{100}^{\min }(1) \approx 9 \cdot 10^{-161}, x_{100}^{\min }(10) \approx-5 \cdot 10^{-60}, \\
x_{100}^{\min }(20) \approx 2 \cdot 10^{-29}, x_{100}^{\min }(30) \approx-1.5 \cdot 10^{-11}, x_{100}^{\min }(38.1) \approx-0.639 .
\end{gathered}
$$




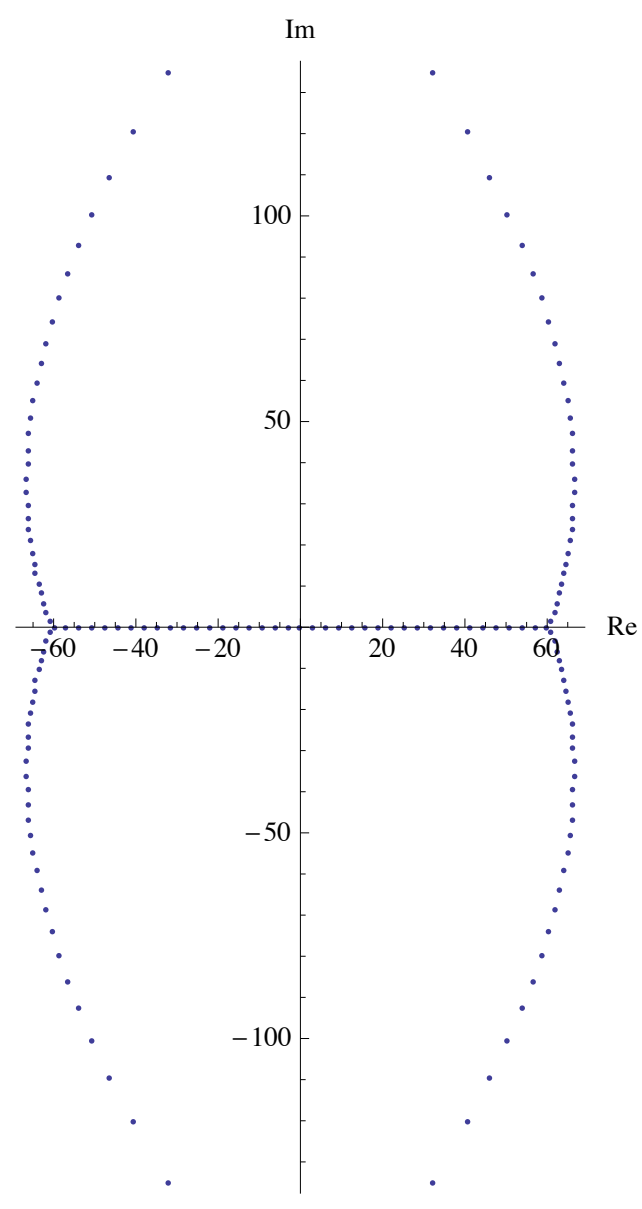

Figure 9: The complex zeros of the polynomial $f_{40}$ defined in 10 .

We add that any approximate real number above can exactly be represented as a root of an integer polynomial of degree 200, whose coefficients can typically be written altogether by approximately 76000 digits. So to display the graph of $x_{n}^{\min }(\cdot)$ in a meaningful way, some scaling has to be applied. For fixed $n$ values, Figures 10 and 11 actually display the curves

$$
y \mapsto \operatorname{sign}\left(x_{n}^{\min }(y)\right) \cdot \frac{-1}{\log _{10}\left|x_{n}^{\min }(y)\right|}
$$

with $y$ values measured along the vertical axis (corresponding to the imaginary axis) and function values along the horizontal one (corresponding to the real axis). Since for each $n$ and $y$ value we now have $\left|x_{n}^{\min }(y)\right|<1$, the sign correction ensures that a point on the figure is in the open left half-plane (or right half-plane) if and only if $\operatorname{sign}\left(x_{n}^{\min }(y)\right)=-1$ (or 1 ). The 
intersection points of the graph of $x_{n}^{\min }(\cdot)$ and the vertical axis correspond to the endpoints of the shaded intervals in Figure 7. The curve segments bounded by the vertical red dashed lines (placed at $\pm 10^{-16}$ ) correspond to stability region boundaries that are "invisible" by using machine precision. As for the vertical (black or red) dotted lines in Figure 11, they measure the amplitude of the oscillations (that is, the local extrema) of (13) in the interval $\left[-10^{-16}, 10^{-16}\right]$, and are found approximately at

$$
-10^{-19},-10^{-30},-10^{-45},-10^{-72}, 10^{-90}, 10^{-55}, 10^{-38}, 10^{-24} .
$$

\section{Semi-disks contained in $\mathcal{S}_{n}$ in the left half-plane}

Results of this section are formulated mostly in terms of the scaled stability region $\mathcal{S}_{n}$.

First we prove a theorem giving a necessary and sufficient condition for a small enough semi-disk in the left half-plane and centered at the origin to be contained in $\mathcal{S}_{n}$. Then we present the radius of the maximal such semi-disks for $n \leq 20$. Finally we show some figures concerning the radial slices of $\mathcal{S}_{n} \cap\{\Re \leq 0\}$.

Theorem 5.1. Let $n$ be a positive integer. Then

$$
\exists \varrho>0: \quad D_{\varrho} \cap\{\Re \leq 0\} \subset \mathcal{S}_{n}
$$

if and only if $n \equiv 0 \bmod 4$ or $n \equiv 3 \bmod 4$.

Proof. Corollary 4.2 establishes that $D_{\varrho} \cap\{\Re \leq 0\} \not \subset \mathcal{S}_{n}$ for any $\varrho>0$ and $n \equiv 1 \bmod 4$ or $n \equiv 2 \bmod 4$, so to finish the proof we show that a sufficiently small closed left semi-disk centered at the origin is contained in $\mathcal{S}_{n}$ for $n \equiv 0 \bmod 4$ or $n \equiv 3 \bmod 4$.

To this end we fix an $n \geq 1$ with $n \equiv 0 \bmod 4$ or $n \equiv 3 \bmod 4$ and notice that

$$
\left|\sum_{k=0}^{n} \frac{(x+i y)^{k}}{k !}\right|^{2}=1+2 x+P_{n}(x, y),
$$

where $x, y \in \mathbb{R}$ and the polynomial $P_{n}$ has the form

$$
P_{n}(x, y)=\sum_{k \geq 0, \ell \geq 0, k+\ell \geq 2, \ell \text { is even }} a_{k, \ell} x^{k} y^{\ell}
$$

with some real coefficients $a_{k, \ell}$. Then with $x=r \cos \varphi$ and $y=r \sin \varphi\left(r \geq 0, \frac{\pi}{2} \leq \varphi \leq \pi\right.$; due to symmetry, only the upper left quadrant is considered) we have

$$
\partial_{\varphi}\left(\left|\sum_{k=0}^{n} \frac{(r \exp (i \varphi))^{k}}{k !}\right|^{2}\right)=(r \sin \varphi)\left(-2+r Q_{n}(r, \cos \varphi, \sin \varphi)\right),
$$




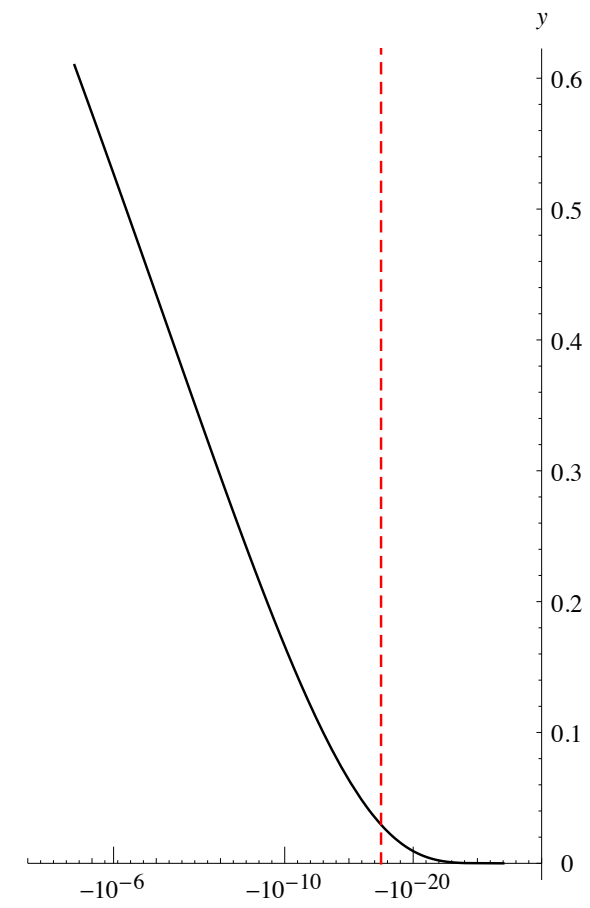

(a)

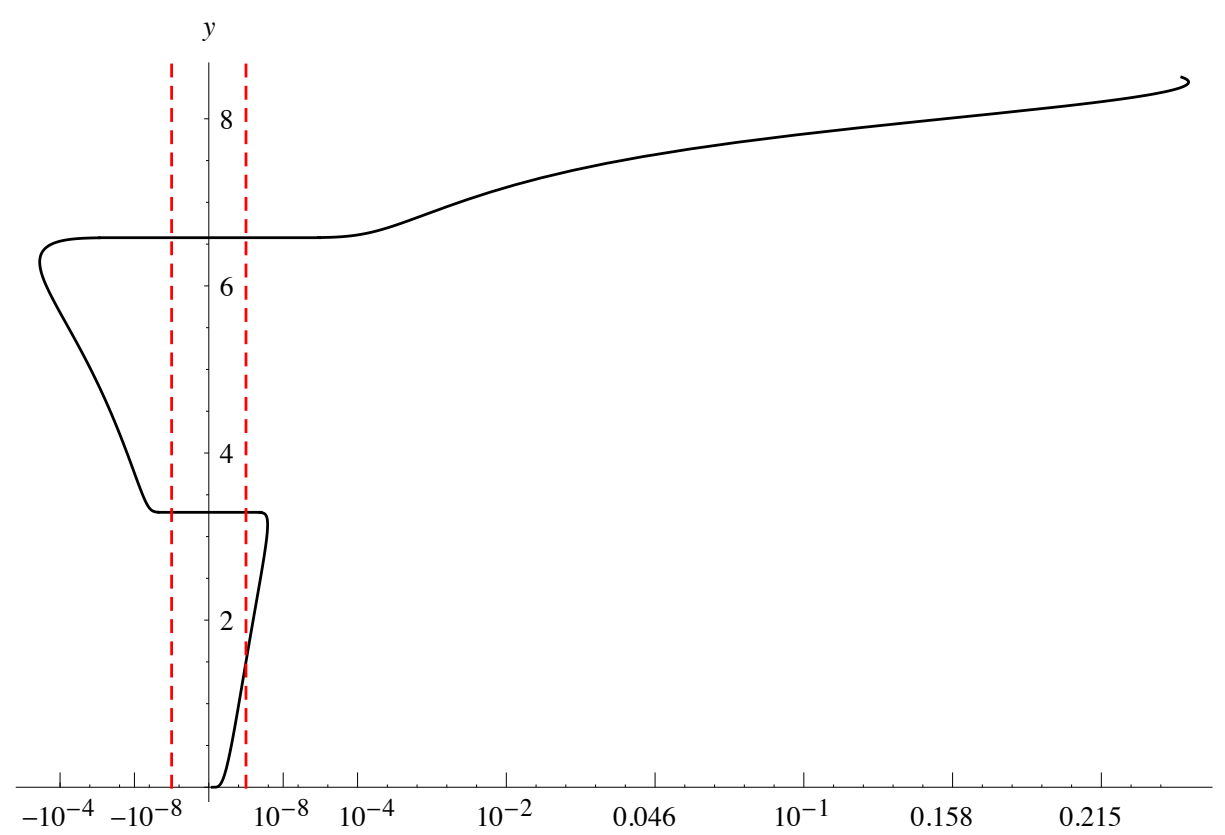

(b)

Figure 10: The boundary curve of $\mathcal{U}_{n}$ closest to the imaginary axis, on an "inverse logarithmic scale", for (a) $n=6$ and (b) $n=20$, see (13). 


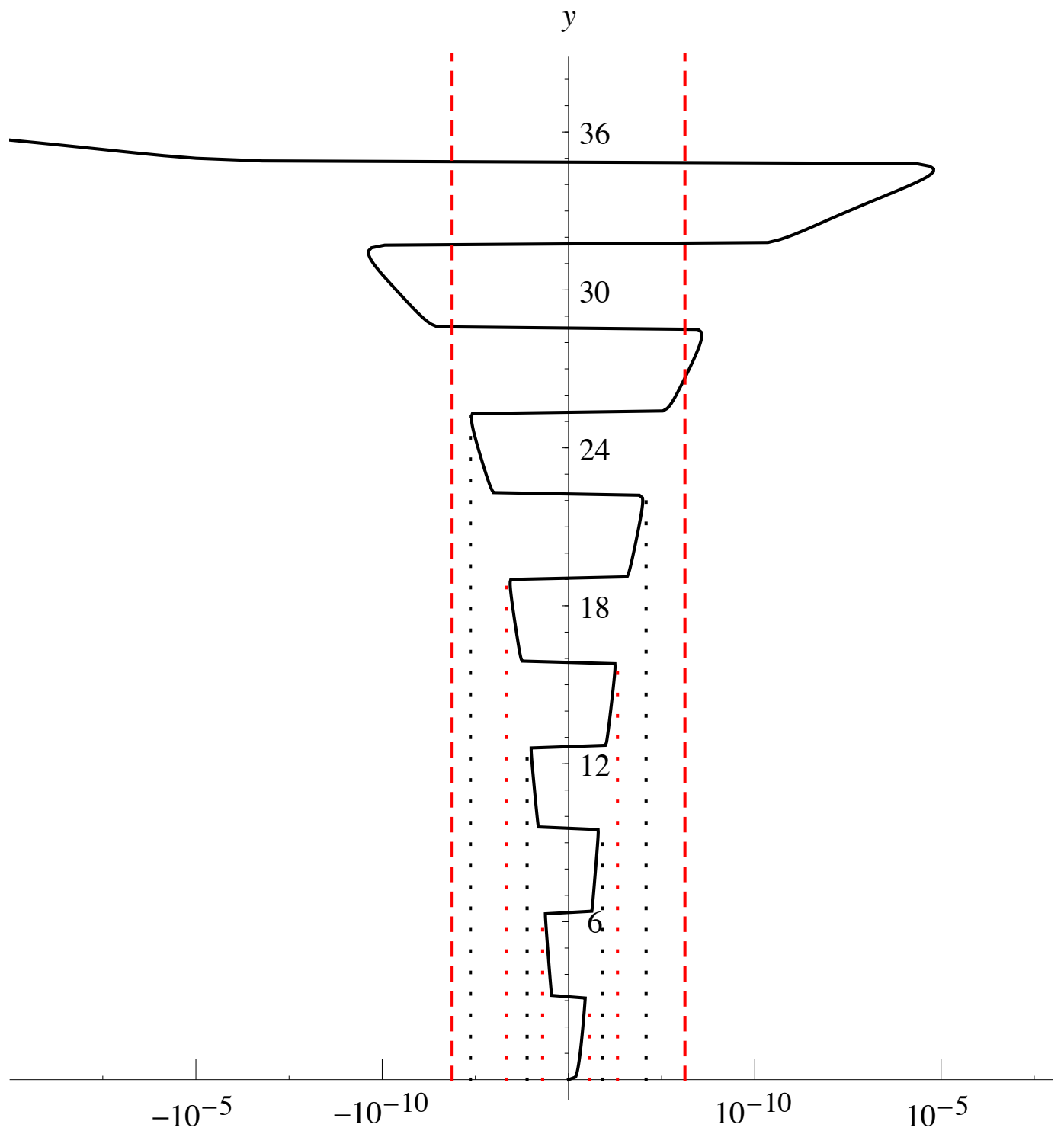

Figure 11: The boundary curve of $\mathcal{U}_{100}$ closest to the imaginary axis, on an "inverse logarithmic scale", see (13).

where $Q_{n}$ is a suitable real polynomial in three variables. Consequently, there exists $r_{n}^{*}>0$ such that for any fixed $0 \leq r \leq r_{n}^{*}$ the function

$$
\left[\frac{\pi}{2}, \pi\right] \ni \varphi \mapsto\left|\sum_{k=0}^{n} \frac{(r \exp (i \varphi))^{k}}{k !}\right|^{2}
$$


is non-increasing, hence its maximal value occurs (for example) at $\varphi=\frac{\pi}{2}$. So by the second part of Corollary 4.2 , for $|z| \leq \min \left(r_{n}^{*}, \varrho_{n}\right)$ and $\Re(z) \leq 0$ we have

$$
\left|\sum_{k=0}^{n} \frac{z^{k}}{k !}\right|^{2} \leq\left|\sum_{k=0}^{n} \frac{(i|z|)^{k}}{k !}\right|^{2} \leq 1
$$

For any $3 \leq n \leq 20$ and $n \equiv 0 \bmod 4$ or $n \equiv 3 \bmod 4$, we have determined the maximal $\varrho_{n}^{*}>0$ radius as an exact algebraic number such that $D_{\varrho_{n}^{*}} \cap\{\Re \leq 0\} \subset \mathcal{S}_{n}$ (see Table 3 ) as follows. It is seen from the definition of $\mathcal{V}_{n}^{+}$(and by taking into account the scaling) that the length of the largest interval in $\mathcal{V}_{n}^{+}$containing 0 is an upper bound on $n \varrho_{n}^{*}$. Let $y_{n, 1}>0$ denote the length of this largest interval. We first exactly determine $y_{n, 1}$ with Mathematica's Reduce (by locating the smallest positive root of the appropriate polynomial in Lemma 4.1), then show (with Reduce again) that no real numbers $x$ and $y$ can satisfy the system

$$
x^{2}+y^{2} \leq\left(y_{n, 1}\right)^{2}, \quad x \leq 0, \quad\left|\sum_{k=0}^{n} \frac{(x+i y)^{k}}{k !}\right|>1,
$$

proving that $\varrho_{n}^{*}=y_{n, 1} / n$. Interestingly, the above simple approach breaks down for $n=4$ : it turns out that $\varrho_{4}^{*}<y_{4,1} / 4$, see Figure 12 .

Thus it seems that the radius of the maximal semi-disk included in $\mathcal{U}_{n}$ tends to $\pi / 2$ for $n \equiv 3 \bmod 4$ and to $\pi$ for $n \equiv 0 \bmod 4$. But it is clear from material in earlier sections that, excepting a small region near the imaginary axis, $\mathcal{U}_{n}$ covers a much larger semi-disk (of radius approximately $n / e$ ). It is interesting to examine by how much the boundary of

$$
\mathcal{S}_{n} \cap\{\Re \leq 0\} \cap\{\Im \geq 0\}
$$

deviates from the asymptotic semi-disk shape indicated by Theorem 1.3 .

For a fixed $n \geq 1$ and some $\varphi \in\left[\frac{\pi}{2}, \pi\right]$, let us define the non-empty set

$$
\mathcal{R}_{n}(\varphi):=\left\{r \in \mathbb{R}: r \geq 0, r e^{i \varphi} \in \mathcal{S}_{n}\right\}
$$

For each $2 \leq n \leq 20$ we determined $\mathcal{R}_{n}(\varphi)$ for approximately 160 different $\varphi \in\left[\frac{\pi}{2}, \pi\right]$ values. These investigations suggest that if a small wedge near the imaginary axis is ignored, then $\mathcal{S}_{n} \cap\{\Re \leq 0\} \cap\{\Im \geq 0\}$ is a starlike set in $\mathbb{C}$ with respect to the origin. In other words, we conjecture that for each $n \geq 1$ there exists a $\varphi_{n} \in\left[0, \frac{\pi}{2}\right)$ with $\varphi_{n} \ll 1$ such that for every $\varphi$ with $\frac{\pi}{2}+\varphi_{n} \leq \varphi \leq \pi$, the set $\mathcal{R}_{n}(\varphi)$ is a compact interval with $\min \left(\mathcal{R}_{n}(\varphi)\right)=0$. For example, the following values of $\varphi_{n}$ seem to be appropriate: $\varphi_{4}=0 ; \varphi_{6}=0$ with 


\begin{tabular}{c|c|c|c}
$n$ & $\varrho_{n}^{*}$ & $n \varrho_{n}^{*}$ & The algebraic degree of $\varrho_{n}^{*}$ \\
\hline 3 & $\sqrt{3} / 3 \approx 0.577$ & $\approx 1.732$ & 2 \\
7 & $\approx 0.252$ & $\approx 1.764$ & 6 \\
11 & $\approx 0.154$ & $\approx 1.701$ & 10 \\
15 & $\approx 0.111$ & $\approx 1.668$ & 14 \\
19 & $\approx 0.086$ & $\approx 1.649$ & 18 \\
\hline 4 & $\approx 0.653$ & $\approx 2.615$ & 24 \\
8 & $\approx 0.424$ & $\approx 3.395$ & 6 \\
12 & $\approx 0.281$ & $\approx 3.379$ & 10 \\
16 & $\approx 0.207$ & $\approx 3.324$ & 14 \\
20 & $\approx 0.164$ & $\approx 3.290$ & 18 \\
\hline
\end{tabular}

Table 3: For $3 \leq n \leq 20$, the table contains the maximal $\varrho_{n}^{*}$ values (whenever they are positive) such that $D_{\varrho_{n}^{*}} \cap\{\Re \leq 0\} \subset \mathcal{S}_{n}$. Instead of listing exact algebraic numbers (apart from the first row, and with degrees given in the last column), the values of $\varrho_{n}^{*}$ are rounded down. For convenience, the maximal inner radius for each unscaled stability region is also given (as $n \varrho_{n}^{*}$ ). The exceptional $n=4$ case is displayed in Figure 12 .

$\mathcal{R}_{6}(\pi / 2)=\{0\}$ but $\mathcal{R}_{6}(\pi / 2+\varepsilon$ ) (for any $0<\varepsilon \leq \pi / 2$ ) being a non-degenerate interval; and there is a suitable $\varphi_{20}$ already in $\left(0,5 \cdot 10^{-5}\right)$. Figure 13 shows the graphs of

$$
\left[\frac{\pi}{2}+\varphi_{n}, \pi\right] \ni \varphi \mapsto \max \left(\mathcal{R}_{n}(\varphi)\right)
$$

for $n=4, n=6$, and $n=20$, with linear interpolation between the approximately 160 different $\varphi$ values in each case. Notice that-for a particular $n$ - the value $\max _{\pi / 2 \leq \varphi \leq \pi}\left(\max \left(\mathcal{R}_{n}(\varphi)\right)\right)$ is found in Table 2, Meanwhile, $\min _{\pi / 2 \leq \varphi \leq \pi}\left(\max \left(\mathcal{R}_{4}(\varphi)\right)\right)$ corresponds to $\varrho_{4}^{*}$ in Table 3; also compare the orange curve in Figure 13 and the dashed brown curve in Figure 12. As for the brown curve in Figure 13 , $\min _{\pi / 2 \leq \varphi \leq \pi}\left(\max \left(\mathcal{R}_{6}(\varphi)\right)\right)=0$, in accordance with the fact that $\mathcal{V}_{6}^{+}=\{0\}$ (see Figure 6). Finally, as for the black curve in Figure 13, the scaled function value around $20 \cdot 0.445 \approx 8.9$ corresponding to $\varphi \approx \pi / 2+\varphi_{20}$ is the highest point of the upper shaded rectangle in Figure 6; the highest point of the lower shaded rectangle is $20 \varrho_{20}^{*}$ in Table 3 ,

\section{Auxiliary lemmas}

Below we prove some additional results that were referenced and used in earlier sections. 


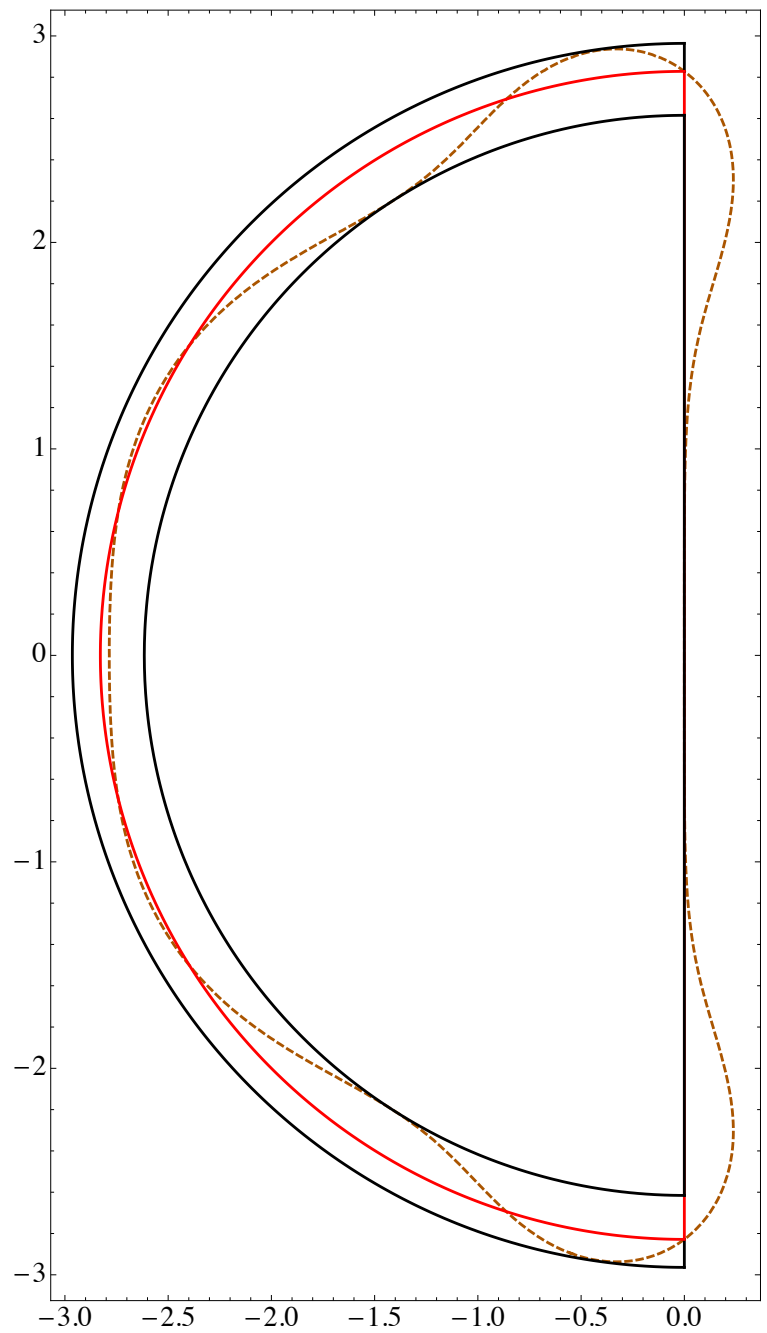

Figure 12: The figure shows the smallest left semi-disk (outer black curve) that contains / the largest left semi-disk (inner black curve) that is contained in $\mathcal{U}_{4}$ (dashed brown curve). The red semi-disk has radius $y_{4,1}=\sqrt{8}$ (notice that $\mathcal{V}_{4}^{+}$is connected, so $y_{4,1}=\max \left(\mathcal{V}_{4}^{+}\right)$); see the corresponding row of Table 3 as well.

Lemma 6.1. For any $n \in \mathbb{N}^{+}$we have $\left(\frac{n}{e}\right)^{n} \sqrt{2 \pi n}<n ! \leq e\left(\frac{n}{e}\right)^{n} \sqrt{n}$.

Proof. The proof is a standard monotonicity argument, hence omitted here.

In the next lemma and later, we make use of the Lambert $W$ function (a.k.a. ProductLog 


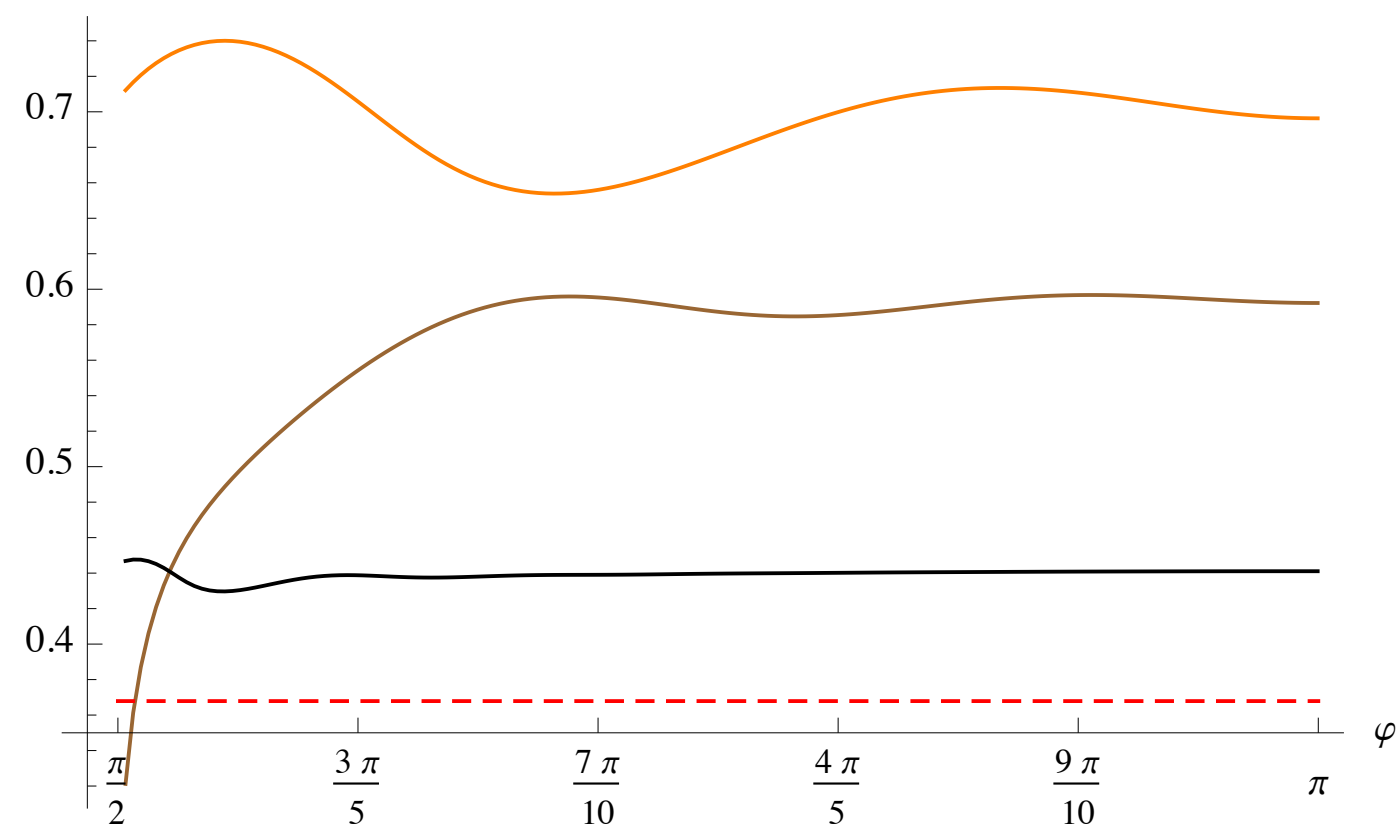

Figure 13: The extent of $\mathcal{S}_{n}$ as a function of the angle $\varphi$ (see (14) and (15)). The orange, brown and black curves correspond to $n=4, n=6$ and $n=20$, respectively. The dashed red line is placed at $1 / e$.

in Mathematica): recall that for $x \geq-\frac{1}{e}$, there is a unique $W(x) \geq-1$ such that

$$
x=W(x) e^{W(x)} .
$$

Lemma 6.2. The set $\Sigma_{1} \subset \mathbb{C}$ is strictly convex.

Proof. By identifying $\mathbb{C}$ with $\mathbb{R}^{2}$, we see that

$$
\Sigma_{1}=\left\{(x, y) \in \mathbb{R}^{2}:-W(1 / e) \leq x \leq 1,-\sqrt{e^{2 x-2}-x^{2}} \leq y \leq \sqrt{e^{2 x-2}-x^{2}}\right\} .
$$

The proof is finished by checking that $\left(\sqrt{e^{2 x-2}-x^{2}}\right){ }^{\prime \prime}<0$ for $-W(1 / e)<x<1$.

Remark 6.3. The value of $-W(1 / e)$ is $\approx-0.278464543$.

Lemma 6.4. The inclusion $D_{1 / 4} \subset \Sigma_{1}$ holds.

Proof. Since $|z| \leq 1 / 4$ implies $\Re(z) \geq-1 / 4$, we have $\left|z e^{1-z}\right| \leq \frac{1}{4} e^{5 / 4}<1$. 
Lemma 6.5. We have $C_{1 / e} \cap \Sigma_{1}=\{ \pm i / e\}$. On the other hand, for any $\varrho>\frac{1}{e}, C_{\varrho} \cap \Sigma_{1}=\varnothing$ and

$$
\left|C_{\varrho}, \Sigma_{1}\right| \geq \frac{\varrho e-1}{\sqrt{e^{2}+1}}
$$

Proof. The intersections $C_{\varrho} \cap \Sigma_{1}$ (for $\varrho \geq \frac{1}{e}$ ) are determined by using the explicit representation of $\Sigma_{1}$ given in the proof of Lemma 6.2 combined with the inequality

$$
\sqrt{x^{2}+\left(\sqrt{e^{2 x-2}-x^{2}}\right)^{2}} \leq \frac{1}{e}
$$

valid for $-W(1 / e) \leq x \leq 0$, and noticing that "=" in " $\leq$ " above holds precisely for $x=0$. As for estimating the distance between the sets $C_{\varrho}$ and $\Sigma_{1}$ (viewed as subsets of $\mathbb{R}^{2}$ ), let us fix some $\varrho>\frac{1}{e}$ and $\varphi \in\left[\frac{\pi}{2}, \frac{3 \pi}{2}\right]$, and consider the point $(\varrho \cos \varphi, \varrho \sin \varphi) \in C_{\varrho}$, depicted as point A on Figure 14. Due to symmetry, we can assume $\varphi \in\left[\frac{\pi}{2}, \pi\right]$. Then the line passing through point $\mathbf{A}$ and the origin intersects $\Sigma_{1}$ at point $\mathbf{B}$. We consider the tangent line to $\Sigma_{1}$ at $\mathbf{B}$. The closest point on this tangent line to $\mathbf{A}$ is point $\mathbf{C}$. Since $\Sigma_{1}$ is convex, the distance $|\mathbf{A}, \mathbf{C}|$ is a lower estimate for $\left|\mathbf{A}, \Sigma_{1}\right|$. We keep $\varrho$ fixed, but vary $\varphi$, so $|\mathbf{A}, \mathbf{C}| \equiv|\mathbf{A}(\varphi), \mathbf{C}(\varphi)|$. Then clearly,

$$
\left|C_{\varrho}, \Sigma_{1}\right| \geq \inf _{\varphi \in\left[\frac{\pi}{2}, \pi\right]}|\mathbf{A}(\varphi), \mathbf{C}(\varphi)|
$$

In Step 1 below, we show that for $\frac{\pi}{2}<\varphi<\pi$ we have

$$
|\mathbf{A}(\varphi), \mathbf{C}(\varphi)|=\frac{\left|W\left(-\frac{\cos \varphi}{e}\right)+1\right|\left|W\left(-\frac{\cos \varphi}{e}\right)+\varrho \cos \varphi\right|}{\sqrt{\left(W\left(-\frac{\cos \varphi}{e}\right)+\cos ^{2} \varphi\right)^{2}+\cos ^{2} \varphi \sin ^{2} \varphi}}
$$

Obviously, the function $\varphi \mapsto|\mathbf{A}(\varphi), \mathbf{C}(\varphi)|$ is continuous at $\varphi=\frac{\pi}{2}$ and $\varphi=\pi$ as well.

On the other hand, we show in Step 2 below that $\left(\frac{\pi}{2}, \pi\right) \ni \varphi \mapsto|\mathbf{A}(\varphi), \mathbf{C}(\varphi)|$ is strictly increasing, and

$$
\lim _{\varphi \rightarrow \frac{\pi}{2}^{+}}|\mathbf{A}(\varphi), \mathbf{C}(\varphi)|=\frac{\varrho e-1}{\sqrt{e^{2}+1}}
$$

Consequently, $\left|C_{\varrho}, \Sigma_{1}\right| \geq \inf _{\varphi \in\left[\frac{\pi}{2}, \pi\right]}|\mathbf{A}(\varphi), \mathbf{C}(\varphi)|=\frac{\varrho e-1}{\sqrt{e^{2}+1}}$, so the proof of the lemma is finished by proving Steps 1 and 2 .

Step 1. Let us fix some $\varrho>\frac{1}{e}$ and $\frac{\pi}{2}<\varphi<\pi$. We see from the construction that the coordinates of $\mathbf{B}=(x, y)$ satisfy $y=\sqrt{e^{2 x-2}-x^{2}}$ and $y=x \tan \varphi$, so (by using $\tan ^{2} \varphi+1=$ $\left.\frac{1}{\cos ^{2} \varphi}\right)$ we have $\left|\frac{x}{\cos \varphi}\right|=\frac{x}{\cos \varphi}=e^{x-1}$, that is, $x=-W\left(-\frac{\cos \varphi}{e}\right)$. This yields

$$
\mathbf{B}=\left(-W\left(-\frac{\cos \varphi}{e}\right),-W\left(-\frac{\cos \varphi}{e}\right) \tan \varphi\right)
$$


so the tangent line to $\Sigma_{1}$ at $\mathbf{B}$ has slope

$$
\begin{gathered}
\left.\left(\sqrt{e^{2 x-2}-x^{2}}\right)^{\prime}\right|_{x=-W\left(-\frac{\cos \varphi}{e}\right)}= \\
\frac{e^{-2 W\left(-\frac{\cos \varphi}{e}\right)-2}+W\left(-\frac{\cos \varphi}{e}\right)}{\sqrt{e^{-2 W\left(-\frac{\cos \varphi}{e}\right)-2}-W\left(-\frac{\cos \varphi}{e}\right)^{2}}}=-\frac{1}{\sin \varphi \cos \varphi}\left(W\left(-\frac{\cos \varphi}{e}\right)+\cos ^{2} \varphi\right) .
\end{gathered}
$$

Now we see that the equation for the line passing through points $\mathbf{B}$ and $\mathbf{C}$ can be written as $a x+b y+c=0$ with

$$
\begin{gathered}
a=-\frac{1}{\sin \varphi \cos \varphi}\left(W\left(-\frac{\cos \varphi}{e}\right)+\cos ^{2} \varphi\right), \\
b=-1
\end{gathered}
$$

and

$$
\begin{gathered}
c=-W\left(-\frac{\cos \varphi}{e}\right) \tan \varphi-\frac{1}{\sin \varphi \cos \varphi}\left(W\left(-\frac{\cos \varphi}{e}\right)+\cos ^{2} \varphi\right) W\left(-\frac{\cos \varphi}{e}\right)= \\
-\frac{1}{\sin \varphi \cos \varphi} W\left(-\frac{\cos \varphi}{e}\right)\left(W\left(-\frac{\cos \varphi}{e}\right)+1\right) .
\end{gathered}
$$

Therefore, the distance from point $\mathbf{A}=(\varrho \cos \varphi, \varrho \sin \varphi)$ to this line is given by

$$
\frac{|a \varrho \cos \varphi+b \varrho \sin \varphi+c|}{\sqrt{a^{2}+b^{2}}}=\frac{\left|W\left(-\frac{\cos \varphi}{e}\right)+1\right|\left|W\left(-\frac{\cos \varphi}{e}\right)+\varrho \cos \varphi\right|}{\sqrt{\left(W\left(-\frac{\cos \varphi}{e}\right)+\cos ^{2} \varphi\right)^{2}+\cos ^{2} \varphi \sin ^{2} \varphi}}
$$

Step 2. It is convenient to set $w \equiv w(\varphi):=W\left(-\frac{\cos \varphi}{e}\right)$. Then $w$ is a strictly increasing bijection, mapping $\left[\frac{\pi}{2}, \pi\right]$ onto $\left[0, W\left(\frac{1}{e}\right)\right]$. For $\frac{\pi}{2}<\varphi<\frac{e}{<}$, that is for $0<w<W\left(\frac{1}{e}\right)$, we

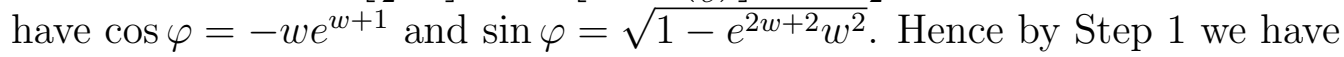

$$
|\mathbf{A}(\varphi), \mathbf{C}(\varphi)|=\frac{|w+1|\left|1-\varrho e^{w+1}\right|}{\sqrt{e^{2 w+2}(2 w+1)+1}} .
$$

Now $w+1>w>0$, and for $\varrho>\frac{1}{e}$ one has $1-\varrho e^{w+1}<0$, so

$$
|\mathbf{A}(\varphi), \mathbf{C}(\varphi)|=\frac{(w+1)\left(\varrho e^{w+1}-1\right)}{\sqrt{e^{2 w+2}(2 w+1)+1}} .
$$


On the other hand, it is trivial that

$$
\partial_{w}\left(\frac{(w+1)\left(\varrho e^{w+1}-1\right)}{\sqrt{e^{2 w+2}(2 w+1)+1}}\right)=\frac{e^{2 w+2}\left(2 w^{2}+2 w+1\right)+\varrho e^{3 w+3} w+\varrho e^{w+1}(w+2)-1}{\left(e^{2 w+2}(2 w+1)+1\right)^{3 / 2}}>0
$$

for any $\varrho>\frac{1}{e}$ and $w>0$, so $\left(\frac{\pi}{2}, \pi\right) \ni \varphi \mapsto|\mathbf{A}(\varphi), \mathbf{C}(\varphi)|$ is strictly increasing, and

$$
\lim _{\varphi \rightarrow \frac{\pi}{2}^{+}}|\mathbf{A}(\varphi), \mathbf{C}(\varphi)|=\lim _{w \rightarrow 0^{+}} \frac{(w+1)\left(\varrho e^{w+1}-1\right)}{\sqrt{e^{2 w+2}(2 w+1)+1}}=\frac{\varrho e-1}{\sqrt{e^{2}+1}} .
$$

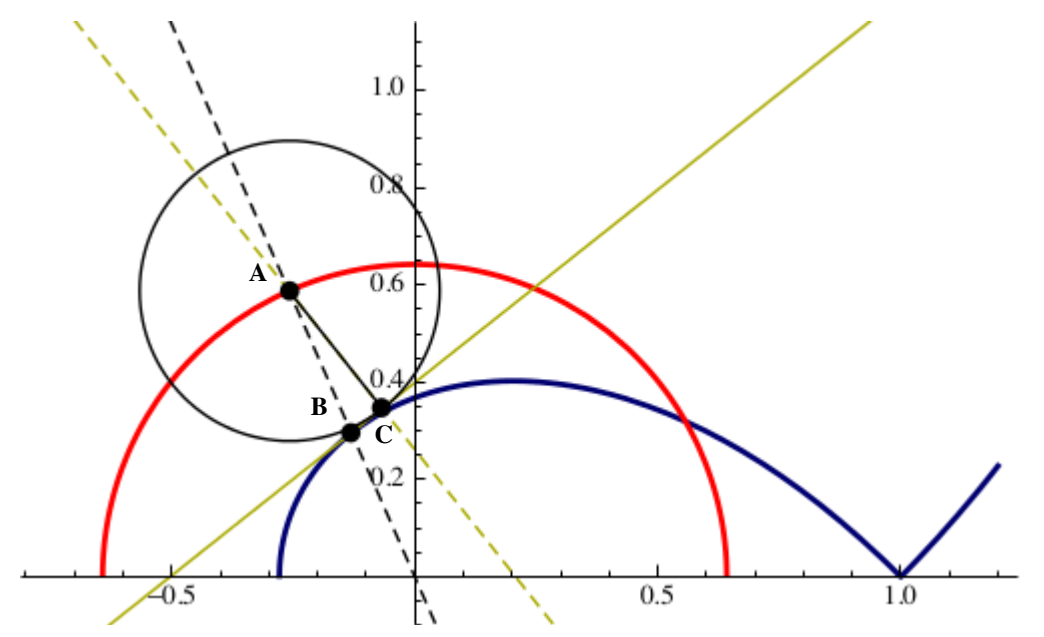

Figure 14: The construction used in the proof of Lemma 6.5.

Remark 6.6. For $\varrho>\frac{1}{e}$ we have $i \varrho \in C_{\varrho}$ and $\frac{i}{e} \in \Sigma_{1}$, so

$$
\left|C_{\varrho}, \Sigma_{1}\right| \leq \varrho-\frac{1}{e}
$$

Lemma 6.7. For any $\sigma>0$ we have

$$
\inf \left\{\left|\frac{w}{w-1}\right|: w \in \mathbb{C},|w| \geq \sigma\right\}=\frac{\sigma}{1+\sigma} .
$$

On the other hand, we have

$$
\forall \varrho \in\left[0.95, \frac{1+\sqrt{1+e^{2}}}{e}\right]: \quad \sup \left\{\left|\frac{w}{w-1}\right|: w \in \mathbb{C},\left|w, \Sigma_{1}\right| \geq \delta_{\varrho}, \Re(w) \leq \delta_{\varrho}\right\} \leq 1,
$$


and

$$
\forall \varrho \in\left(\frac{1+\sqrt{1+e^{2}}}{e}, 2\right]: \quad \sup \left\{\left|\frac{w}{w-1}\right|: w \in \mathbb{C},\left|w, \Sigma_{1}\right| \geq \delta_{\varrho}, \Re(w) \leq \delta_{\varrho}\right\} \leq 1.39,
$$

where

$$
\delta_{\varrho}:=\frac{\varrho e-1}{2 \sqrt{e^{2}+1}}
$$

Proof. For (17), we rewrite the expression as

$$
\inf \left\{\sqrt{x^{2}+y^{2}} / \sqrt{(x-1)^{2}+y^{2}}: x, y \in \mathbb{R}, \sqrt{x^{2}+y^{2}} \geq \sigma\right\},
$$

and see that $\sqrt{(-\sigma)^{2}+0^{2}} / \sqrt{(-\sigma-1)^{2}+0^{2}}=\sigma /(1+\sigma)$. On the other hand, we directly verify that

$$
\frac{\sqrt{x^{2}+y^{2}}}{\sqrt{(x-1)^{2}+y^{2}}}<\frac{\sigma}{1+\sigma}, \quad \sqrt{x^{2}+y^{2}} \geq \sigma
$$

has no real $x, y$ solutions for any $\sigma>0$.

As for $(18)$, we have $0<\delta_{\varrho} \leq 1 / 2$ for the given $\varrho$ values, so

$$
\sup \left\{\left|\frac{w}{w-1}\right|: w \in \mathbb{C},\left|w, \Sigma_{1}\right| \geq \delta_{\varrho}, \Re(w) \leq \delta_{\varrho}\right\} \leq \sup \left\{\left|\frac{w}{w-1}\right|: w \in \mathbb{C}, \Re(w) \leq \frac{1}{2}\right\}=1 .
$$

Finally, for $(19)$, we notice that, due to $1 \in \Sigma_{1},\left|w, \Sigma_{1}\right| \geq \delta_{\varrho}$ implies $|w, 1| \geq \delta_{\varrho}$, so the supremum is estimated from above by $\sup \left\{\left|\frac{w}{w-1}\right|: w \in \mathbb{C},|w-1| \geq \delta_{\varrho}, \Re(w) \leq \delta_{\varrho}\right\}$. Elementary computation shows that for each $\varrho$ in the given range and for $w \in \mathbb{C}$ with $|w-1| \geq \delta_{\varrho}$ and $\Re(w) \leq \delta_{\varrho},\left|\frac{w}{w-1}\right|$ is maximal when $|w-1|=\delta_{\varrho}=\Re(w)$. For such $w$, the maximal value of $\left|\frac{w}{w-1}\right|$ is

$$
\frac{\sqrt{e^{2}\left(\varrho^{2}-4\right)+2 e\left(2 \sqrt{1+e^{2}}-1\right) \varrho-4 \sqrt{1+e^{2}}-3}}{\varrho e-1}<1.39 .
$$

\section{References}

[1] P. Bleher and R. Mallison, Jr. Zeros of sections of exponential sums. Int. Math. Res. Not., Art. ID 38937:1-49, 2006. 
[2] J. D. Buckholtz. Concerning an approximation of Copson. Proc. Amer. Math. Soc., 14:564-568, 1963.

[3] J. D. Buckholtz. A characterization of the exponential series. Amer. Math. Monthly, 73(4, part II):121-123, 1966.

[4] A. J. Carpenter, R. S. Varga, and J. Waldvogel. Asymptotics for the zeros of the partial sums of $e^{z}$. I. Rocky Mountain J. Math., 21(1):99-120, 1991.

[5] J. Dieudonné. Sur les zéros des polynomes-sections de $e^{x}$. Bull. Sci. Math., 70:333-351, 1935.

[6] R. B. Gardner and N. K. Govil. Some generalizations of the Eneström-Kakeya theorem. Acta Mathematica Hungarica, 74(1-2):125-134, 1997.

[7] R. Jeltsch and O. Nevanlinna. Stability of explicit time discretizations for solving initial value problems. Numer. Math., 37(1):61-91, 1981.

[8] R. Jeltsch and O. Nevanlinna. Stability and accuracy of time discretizations for initial value problems. Numer. Math., 40(2):245-296, 1982.

[9] M. Kappert. On the zeros of the partial sums of $\cos (z)$ and $\sin (z)$. Numer. Math., 74(4):397-417, 1996.

[10] D. I. Ketcheson, L. Lóczi, and M. Parsani. Propagation of internal errors in explicit Runge-Kutta methods and internal stability of SSP and extrapolation methods. http://arxiv.org/abs/1309.1317.

[11] M. J. Merkle. Inequalities for residuals of power series: a review. Univ. Beograd. Publ. Elektrotehn. Fak. Ser. Mat., 6:79-85, 1995.

[12] D. J. Newman and T. J. Rivlin. The zeros of the partial sums of the exponential function. J. Approximation Theory, 5:405-412, 1972.

[13] D. J. Newman and T. J. Rivlin. Correction to: "The zeros of the partial sums of the exponential function" (J. Approximation Theory 5 (1972), 405-412). J. Approximation Theory, 16(4):299-300, 1976.

[14] I. E. Pritsker and R. S. Varga. The Szegö curve, zero distribution and weighted approximation. Trans. Amer. Math. Soc., 349(10):4085-4105, 1997.

[15] E. B. Saff and R. S. Varga. On the zeros and poles of Padé approximants to $e^{z}$. Numer. Math., 25(1):1-14, 1975/76. 
[16] E. B. Saff and R. S. Varga. Zero-free parabolic regions for sequences of polynomials. SIAM J. Math. Anal., 7(3):344-357, 1976.

[17] G. Szegő. Über eine Eigenshaft der Exponentialreihe. Sitzungsber. Berl. Math. Ges., (23):50-64, 1924.

[18] E. C. Titchmarsh. The Theory of Functions (Second Edition). Oxford University Press, 1939.

[19] R. S. Varga and A. J. Carpenter. Asymptotics for the zeros of the partial sums of $e^{z}$. II. In Computational methods and function theory (Valparaiso, 1989), volume 1435 of Lecture Notes in Math., pages 201-207. Springer, Berlin, 1990.

[20] R. S. Varga and A. J. Carpenter. Zeros of the partial sums of $\cos (z)$ and $\sin (z)$. I. Numer. Algorithms, 25:363-375, 2000.

[21] R. S. Varga and A. J. Carpenter. Zeros of the partial sums of $\cos (z)$ and $\sin (z)$. II. Numer. Math., 90(2):371-400, 2001.

[22] R. S. Varga and A. J. Carpenter. Zeros of the partial sums of $\cos (z)$ and $\sin (z)$. III. Appl. Numer. Math., 60(4):298-313, 2010.

[23] R. S. Varga, A. J. Carpenter, and B. W. Lewis. The dynamical motion of the zeros of the partial sums of $e^{z}$, and its relationship to discrepancy theory. Electron. Trans. Numer. Anal., 30:128-143, 2008.

[24] A. R. Vargas. Zeros of sections of some power series. MSc Thesis, Dalhousie University, Halifax, 2012.

[25] P. Walker. The zeros of the partial sums of the exponential series. Amer. Math. Monthly, 110(4):337-339, 2003.

[26] G. Wanner, E. Hairer, and S. P. Nørsett. Order stars and stability theorems. BIT Numerical Mathematics, 18(4):475-489, December 1978.

[27] C. Y. Yıldırım. On the tails of the exponential series. Canad. Math. Bull., 37(2):278-286, 1994.

[28] S. M. Zemyan. On the Zeroes of the Nth Partial Sum of the Exponential Series. Amer. Math. Monthly, 112(10):891-909, 2005. 